\title{
AGN heating and ICM cooling in the HIFLUGCS sample of galaxy clusters ${ }^{\star}$
}

\author{
R. Mittal ${ }^{1,2}$, D. S. Hudson ${ }^{1}$, T. H. Reiprich ${ }^{1}$, and T. Clarke ${ }^{3,4}$ \\ 1 Argelander-Institut für Astronomie, Auf dem Hügel 71, 53121 Bonn, Germany \\ e-mail:rmittal@astro.uni-bonn.de \\ 2 Max-Planck-Institute für Radioastronomie, Auf dem Hügel 69, 53121 Bonn, Germany \\ 3 Naval Research Laboratory, Code 7213, 4555 Overlook Ave. SW, Washington, D. C. 20375, USA \\ ${ }^{4}$ Interferometrics Inc., 13454 Sunrise Valley Drive, Suite 240, Herndon, VA 20171, USA
}

Received 20 August 2008 / Accepted 12 January 2009

\section{ABSTRACT}

\begin{abstract}
Active galactic nuclei (AGN) at the center of galaxy clusters with gas cooling times that are much shorter than the Hubble time have emerged as heating agents powerful enough to prevent further cooling of the intracluster medium (ICM). We carried out an intensive study of the AGN heating-ICM cooling network by comparing various cluster parameters to the integrated radio luminosity of the central AGN, $L_{\mathrm{R}}$, defined as the total synchrotron power between $10 \mathrm{MHz}$ and $15 \mathrm{GHz}$. This study is based on the HIFLUGCS sample comprising the $64 \mathrm{X}$-ray brightest galaxy clusters. We adopted the central cooling time, $t_{\text {cool }}$, as the diagnostic to ascertain cooling properties of the HIFLUGCS sample and classify clusters with $t_{\text {cool }}<1 \mathrm{Gyr}$ as strong cool-core (SCC) clusters, with $1 \mathrm{Gyr}<$ $t_{\text {cool }}<7.7 \mathrm{Gyr}$ as weak cool-core (WCC) clusters and with $t_{\text {cool }}>7.7 \mathrm{Gyr}$ as non-cool-core (NCC) clusters. We find 48 out of 64 clusters $(75 \%)$ contain cluster center radio sources (CCRS) cospatial with or within $50 h_{71}^{-1} \mathrm{kpc}$ of the X-ray peak emission. Furthermore, we find that the probability of finding a CCRS increases from $45 \%$ to $67 \%$ to $100 \%$ for NCC, WCC, and SCC clusters, respectively.

We use a total of $\sim 140$ independent radio flux-density measurements, with data at more than two frequencies for more than $54 \%$ of the sources extending below $500 \mathrm{MHz}$, enabling the determination of accurate estimates of $L_{\mathrm{R}}$. We find that $L_{\mathrm{R}}$ in SCC clusters depends strongly on the cluster scale such that more massive clusters harbor more powerful radio AGN. The same trend is observed between $L_{\mathrm{R}}$ and the classical mass deposition rate, $\dot{M}_{\text {classical }}$ in SCC and partly also in WCC clusters, and can be quantified as $L_{\mathrm{R}} \propto \dot{M}_{\text {classical }}^{1.69 \pm .25}$. We also perform correlations of the luminosity for the brightest cluster galaxy, $L_{\mathrm{BCG}}$, close to the X-ray peak in all 64 clusters with $L_{\mathrm{R}}$ and cluster parameters, such as the virial mass, $M_{500}$, and the bolometric X-ray luminosity, $L_{\mathrm{X}}$. To this end, we use the 2MASS $K$-band magnitudes and invoke the near-infrared bulge luminosity-black hole mass relation to convert $L_{\mathrm{BCG}}$ to supermassive black hole mass, $M_{\mathrm{BH}}$. We find a weak correlation between $M_{\mathrm{BH}}$ and $L_{\mathrm{R}}$ for SCC clusters, $L_{\mathrm{R}} \sim M_{\mathrm{BH}}^{4.10 \pm 0.42}$, although with a few outliers. We find an excellent correlation of $L_{\mathrm{BCG}}$ with $M_{500}$ and $L_{\mathrm{X}}$ for the entire sample, the SCC clusters showing a tighter trend in both the cases. We discuss the plausible reasons behind these scaling relations in the context of cooling flows and AGN feedback.

Our results strongly suggest an AGN-feedback machinery in SCC clusters, which regulates the cooling in the central regions. Since the dispersion in these correlations, such as that between $L_{\mathrm{R}}$ and $\dot{M}_{\text {classical }}$ or $L_{\mathrm{R}}$ and $M_{\mathrm{BH}}$, increases in going from SCC to WCC clusters, we conclude there must be secondary processes that work either in conjunction with the AGN heating or independently to counteract the radiative losses in WCC clusters.
\end{abstract}

Key words. cooling flows - galaxies: active - X-rays: galaxies: clusters - radio continuum: galaxies - galaxies: clusters: general

\section{Introduction}

In recent years, heating by active galactic nuclei (AGN) through outflows has gained fundamental importance in the realm of large-scale structure and galaxy formation. Several studies (e.g. Sijacki et al. 2007; Croton et al. 2006; Bower et al. 2006; Scannapieco et al. 2005; Silk \& Rees 1998) have contributed to this comprehensive picture, wherein AGN feedback is considered an attractive solution to several connected problems, such as the high-mass end truncation of galaxy distribution (e.g. Benson et al. 2003) and the absence of cooling-flows in centers of galaxy clusters (e.g. McNamara \& Nulsen 2007). According to these studies, AGN heating at the centers of clusters may likely be

\footnotetext{
* Table 1 is only available in electronic form at http : //www . aanda.org
}

responsible for quenching condensation of the hot intracluster medium onto the cluster galaxies, thereby giving rise to the cutoff at the bright-end of the galaxy luminosity function and also regulating the cooling flows.

Gas in the ICM cools via X-ray emission. In the centers of some clusters, the high density leads to significant loss of energy, such that the gas radiates away all its energy in a short $\left(\ll 1 / H_{0}\right)$ time. In the absence of any heating mechanisms, in order to support the overlying gas and restore hydrostatic equilibrium, there is a steady inflow of gas towards the cluster center, which is often referred to as the classical cooling flow model (Fabian 1994). These so-called cool-core clusters (CC) have centrally peaked $\mathrm{X}$-ray surface-brightness profiles implying gas cooling times orders of magnitude shorter than the age of the cluster. However, (1) the high resolution XMM-Newton RGS spectra of CC clusters have not found the expected amounts of cool gas in their 
cores (e.g. Tamura et al. 2001; Peterson et al. 2001; Kaastra et al. 2001; Peterson et al. 2003; Xu et al. 2002; Sakelliou et al. 2002; Sanders et al. 2008), and; (2) even though the cooling of the ICM is manifested in the form of on-going star formation observed in the brightest cluster galaxy of several clusters (e.g. Mittaz et al. 2001; Allen 1995), it is far below the predicted amount of the star formation rates and CO (e.g. McNamara \& O'Connell 1989; Edge \& Frayer 2003). Additionally, the gas temperature in the central regions as determined from the X-ray spectra of these clusters is much higher than that expected based on the cooling flow model and has been found to drop not much below $40 \%$ the ambient temperature (e.g. Hudson et al. 2008).

Several heating strategies have been proposed to overcome the cooling flow problem. Feedback from supernovae is an important form of heating but has been shown to be sufficient to balance energy losses only in low-luminosity ellipticals with shallower gravitational potentials (Mathews \& Brighenti 2003).

Another heating scenario is thermal conduction which leads to an inward heat flow from the outskirts of the galaxy clusters. Voigt \& Fabian (2004) have shown that even though thermal conduction may provide enough heating to offset cooling in the hotter $(T \gtrsim 5 \mathrm{keV})$ part of the clusters, the central parts of the cooling region remain largely unaffected by this process. Similar to supernovae heating, thermal conduction also has the effect of only slowing down the evolution of intracluster medium by causing the cooling time to increase by a factor of a few (Pope et al. 2005) but leaves the cooling catastrophe inevitable.

In this work, we focus on the self-regulated AGN feedback as the current favored mechanism to explain the dearth of cooling by-products in galaxy clusters (e.g. Voit \& Donahue 2005; Roychowdhury et al. 2004; Churazov et al. 2002; Binney \& Tabor 1995). In this framework, accretion of the cool collapsed intracluster medium (ICM) ignites the central active galactic nucleus, which returns a fraction of the accreted power back to the ICM. The bulk of the energy transfer is believed to happen through mechanical dissipation of the AGN power. The lead evidence comes from the observations of numerous galaxy clusters featuring X-ray deficit low density regions, known as cavities. Such cavities have been observed to correlate spectacularly with radio jets and lobes indicating that they are likely regions emptied of ICM by the expanding radio lobes [e.g. Perseus, Boehringer et al. (1993); Hydra-A, McNamara et al. (2000); A2052, Blanton et al. (2001); A2597, McNamara et al. (2001); A4059, Heinz et al. (2002); A478, Sun et al. (2003); A2029, Clarke et al. (2004); A2199 Gentile et al. (2007)]. The AGN-blown cavities transfer heat to the ICM potentially by generating sound and weak shock waves (Jones et al. 2002; Fabian et al. 2003; Mathews et al. 2006), by doing $p \mathrm{~d} V$ work against the ambient medium and dissipation of cavity enthalpy in the wake of buoyantly rising cavities (e.g. Ruszkowski et al. 2004; Bîrzan et al. 2004). In addition to direct AGN mechanical heating via radio bubbles, there are also alternative proposed mechanisms such as AGN cosmic-ray heating combined with convection (e.g. Chandran \& Rasera 2007) or conduction (e.g. Guo \& Oh 2008; Voit et al. 2008).

Numerous results over the last couple of decades have confirmed that radio-loud AGN dwell preferentially in brightest group and cluster galaxies (BCGs), as opposed to other galaxies of the same stellar mass (von der Linden et al. 2007; Best et al. 2007; Bagchi \& Kapahi 1994; Valentijn \& Bijleveld 1983). It has also been found in these and other studies that the CC clusters are particularly conducive for $\mathrm{cD}$ galaxies which are radioloud, even though the fraction of radio-loud $\mathrm{cD}$ galaxies in $\mathrm{CC}$ clusters varies from study to study ranging from $70 \%$ to $95 \%$
(Burns 1990; Edwards et al. 2007; Dunn \& Fabian 2006). The spread in the fraction between different studies can be attributed to the varying selection criteria used for constructing cluster samples and the use of not so up-to-date X-ray and radio observations. The latter effect may result in the same cluster being identified as a $\mathrm{CC}$ cluster in some works and a non-coolcore (NCC) cluster in others. A few examples being A1650, which based on Einstein observations has been quoted as a NCC cluster by Burns (1990), but which our data, based on highresolution Chandra observations (Hudson et al. 2008), clearly reveal it to have a cool core with a central cooling time of about a gigayear and a predicted mass deposition rate of about $100 M_{\odot} \mathrm{yr}^{-1}$ (also see Donahue et al. 2005). Similarly, A3158 and A3195 have been identified based on low-sensitivity and low-resolution ASCA data as CC clusters by Edwards et al. (2007). Our results imply otherwise; both are merging systems each with a central cooling time longer than 12 Gyr and the expected mass deposition rates being consistent with zero. Despite these inconsistencies, most studies are by and large in agreement with one another and set the average abundance of radio-loud CC clusters in the local Universe to around $80 \%$.

Recent analyses of galaxy clusters have shown that of those CC clusters which require heating, at least $40 \%$ harbor cavities that contain sufficient energy to balance the radiative losses (Rafferty et al. 2008; Nulsen et al. 2006; Rafferty et al. 2006; Dunn \& Fabian 2006). However, the details of the various heating mechanisms set into motion by the central AGN are not clear and are issues currently under investigation. Also, the local conditions in the ICM that lead to a quasi-steady state of gas deposition onto the central regions, and presumably onto the supermassive black hole, and the concomitant AGN heating of the ICM either periodically or continuously remain largely unknown. A parallel model that is emerging to explain the deviation of the observed cluster properties, especially the entropy profiles, from the predictions of the pure cooling model is linked to preheating or entropy injection at incipient stages of cluster formation, even prior to cluster collapse (McCarthy et al. 2007, and references therein). Preheating is entailed by reduction of central densities, hence, central luminosities, which leads to flat cluster entropy profiles. This modification along with post clusterformation processes, namely radiative cooling and gravitational heating, provides a better match to the observed entropy profiles of galaxy clusters. Yet, while preheating may alone account for the differences in entropy profiles in NCC clusters, catastrophic cooling at small radii in CC clusters can still not be bypassed. In order to maintain their entropy profiles at observed levels, one or more additional sources of on-going heating are required.

In this work, we aim to gain a more comprehensive understanding of the AGN-regulated cooling and heating. We scrutinize the ways in which AGN heating is connected to the cooling of the ICM based solely on the total radio (synchrotron) output of the AGN. To achieve this goal, we use a sample of galaxy clusters for which there exist complete radio and X-ray data. We take our analysis further by examining the scaling relations between the BCG near-infrared luminosity and cluster parameters (mass and luminosity). We derive the mass of the supermassive black hole using the near-infrared bulge luminosity-black hole mass relation and inspect whether there is a relation between the black hole mass and AGN radio luminosity. These correlations are made taking into account the possibility of obtaining different relations depending on the cool or non-cool type cluster environment. The improvement over previous analyses lies in the quality of the cluster sample and of the available X-ray and radio observations. 
We describe the sample in Sect. 2, giving details about radio and X-ray data and related quantities in Sect. 2.1 and Sect. 2.2, respectively. The results are presented in Sect. 3, including fractions of $\mathrm{CC}$ and NCC clusters with and without central radio sources in Sect. 3.1, cooling and AGN activity in Sect. 3.2 and correlations of the BCG luminosity with radio and X-ray parameters in Sect. 3.3. We discuss our results in Sect. 4 and end with conclusions in Sect. 5. Throughout this paper, we assume the $\Lambda \mathrm{CDM}$ concordance Universe with $H_{0}=h_{71} 71 \mathrm{~km} \mathrm{~s}^{-1} \mathrm{Mpc}^{-1}$, $\Omega_{\mathrm{m}}=0.27$ and $\Omega_{\Lambda}=0.73$.

\section{Our sample}

The goal of this study is to cross-correlate the cooling activity with the presence of a radio galaxy ${ }^{1}$ cospatial with the peak of the cooling flow region or, synonymously, the peak of the $\mathrm{X}$-ray emission. Further, we want to investigate whether there is a special coupling between the AGN activity and its surrounding hot cluster medium in $\mathrm{CC}$ clusters as compared to NCC clusters. This calls for an objectively selected sample. Samples selected based on what is available in public archives are subject to unknown selection effects ("archive bias"). For example, the fraction of CC clusters in the Chandra archive may be biased higher (or lower) than the same fraction of clusters in the XMM-Newton archive. To this end, we conduct our study based on the largest X-ray flux-limited sample, the HIFLUGCS (Reiprich \& Böhringer 2002) sample, selected from the ROSATAll-Sky Survey outside the Galactic plane using the flux limit, $f_{\mathrm{X}}(0.1-2.4) \mathrm{keV} \geq 2 \times 10^{-11} \mathrm{erg} \mathrm{s}^{-1} \mathrm{~cm}^{-2}$. This sample comprises the $64 \mathrm{X}$-ray brightest clusters and spans a redshift range $0.0037 \leq z \leq 0.2153$ with the mean $\langle z\rangle \sim 0.05$. All 64 clusters have been observed with Chandra and all but one (A2244) have been observed with XMM-Newton to acquire high quality X-ray data. In this paper we make use only of the Chandra analysis because we are interested in the cluster cores and Chandra has currently the best spatial resolution $(0.5)$ of any X-ray telescope. We note that since flux-limited samples are as such biased towards clusters with high or boosted luminosities, this sample might seem to favor CC clusters preferentially over NCC clusters. At any given redshift, CC clusters are more likely to be picked up than the NCC clusters due to their enhanced central luminosities. However, merging clusters present the same bias as cluster merger events entail temporal enhancements in the global luminosities and temperatures (Ricker \& Sarazin 2001). Since merging clusters are mostly NCC clusters (O'Hara et al. 2006; Hudson et al. 2008), this may balance out the former selection bias towards, at least, the strong (to be defined in Sect. 3.1 ) CC clusters. We note that even in the presence of a bias against transition clusters (neither strong CC clusters nor NCC), objectively selected samples, such as HIFLUGCS, can be directly compared to simulated flux-limited samples, at both low $-z$ and high $-z$, and the bias may be calculated.

\subsection{Radio data}

We compiled and in many cases reanalyzed radio observations of all 64 clusters from either literature or archives (VLA, NVSS, VLSS and MOST) to study the radio properties of the centrally located AGN in the HIFLUGCS clusters. The data from the archives were processed in the Astronomical Image Processing Software (AIPS) package provided by NRAO.

\footnotetext{
1 We use the terms "radio galaxy" and "AGN" interchangeably throughout this paper.
}

High-frequency archival radio data $(500 \mathrm{MHz})$ were analyzed using the standard data-reduction procedures within AIPS, wherein the resulting map usually constituted of a single hybrid image. Low-frequency data (330 MHz and $74 \mathrm{MHz}$ ), in addition, were carefully analyzed to remove bad data affected by radio frequency interference (RFI) using the AIPS tasks, SPFLG and TVFLG. In case of pseudo-continuum mode observations, the effects of bandwidth smearing were tackled by keeping the data separate over the spectral channels. And lastly, in order to correct for 3D effects and image degradation due to bright sources far away from the phase-center, we employed the 3D-imaging feature embedded in the AIPS task, IMAGR. This technique of 3D imaging results in a mosaic of "facets", each of which is independently and simultaneously "cleaned" to yield a final map of the entire primary beam.

\subsubsection{Location of a cluster radio source}

Before pursuing the cross-correlation of the global X-ray properties of our sample with the radio properties of cluster central radio sources (CCRSs), we need to establish a criterion based on which to identify central radio sources. Several works have presented evidence for a special relationship between the cooling activity in cluster cores and BCGs located within a certain distance to the X-ray peak. Recently, Edwards et al. (2007) found in their study based on two samples, the NFPS data set, an X-ray selected sample and the C4 catalog, an optically selected sample built from the SDSS, that only those BCGs that lie within $70 h_{71}^{-1} \mathrm{kpc}$ of the X-ray peak of a cooling flow cluster have significant line emission. Even though the optical line emission observed in these BCGs can be inferred either as a signature of AGN activity or star formation, Edwards et al. (2007) also show that $74 \%$ of the strongly emitting BCGs in the SDSS sample, defined as having the $H_{\alpha}$ equivalent width $>2 \AA$, have the diagnostic emitting-line ratios characteristic of AGN activity (with a likely higher fraction for the BCG). Therefore, the formal basis of marking a radio source as "central" in the study presented here was to have the AGN within $50 h_{71}^{-1} \mathrm{kpc}$ of the X-ray peak, a more conservative limit than that proposed by Edwards et al. (2007). It is noteworthy that this cut (as opposed to a more stringent cut of $12 h_{71}^{-1} \mathrm{kpc}$, see below) had actually to be invoked only for four clusters. These four clusters are A3562, A2142, A4038 and A3376 with the X-ray peak and BCG separation as $30.4 h_{71}^{-1} \mathrm{kpc}, 21.8 h_{71}^{-1} \mathrm{kpc}, 14.9 h_{71}^{-1} \mathrm{kpc}$ and $14.2 h_{71}^{-1} \mathrm{kpc}$, respectively. For the rest of the sample, the flagging was straight forward in that the separation between the X-ray peak and the radio active BCG was less than $12 h_{71}^{-1} \mathrm{kpc}$. The $12 h_{71}^{-1} \mathrm{kpc}$ as the yardstick comes from the fact that since the HIFLUGCS clusters span two orders of magnitude in redshift, the Chandra resolution implies varying accuracies with which the X-ray peak may be determined for different clusters and $12 h_{71}^{-1} \mathrm{kpc}$ corresponds to the worst $1-\sigma$ uncertainty. The separation between the BCG and the X-ray peak for all 64 clusters is shown is Fig. 1. Applying this criterion, we find a total of 48 clusters with centrally located radio sources.

\subsubsection{Integrated radio luminosity of a CCRS}

One of the primary concerns in accumulating the radio data was to have a good spectral coverage, particularly, at the low-end of the radio spectrum. Of the $48 \mathrm{CCRSs}, 65 \%$ have radio data below $500 \mathrm{MHz}$ and $46 \%$ have radio data below $80 \mathrm{MHz}$. Lowfrequency radio observations are important to perform a full 


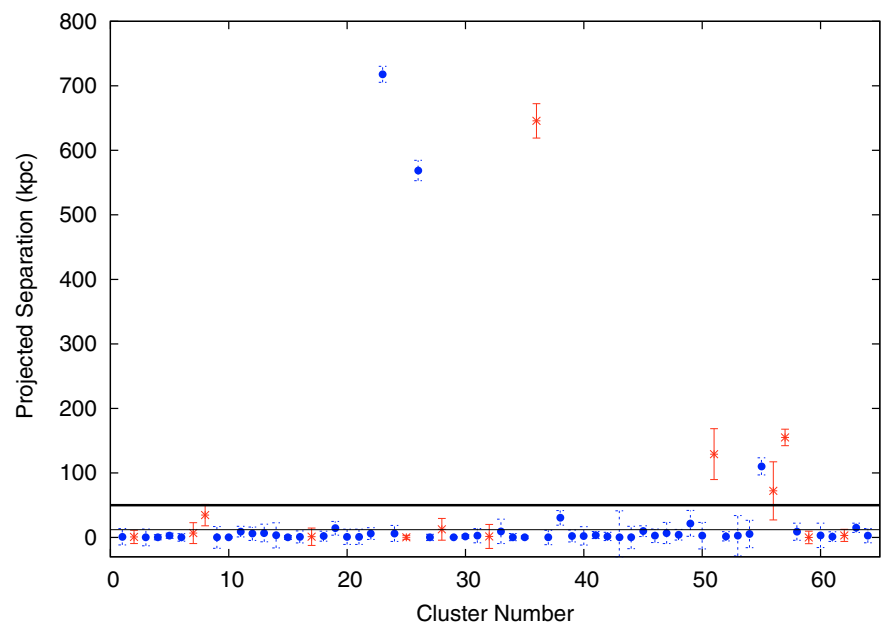

Fig. 1. The separation between the BCG and the X-ray Peak. Plotted on the $X$-axis are numbers assigned to each cluster arbitrarily. The filled circles (blue) represent BCGs which harbor a radio source and the crosses (red) correspond to BCGs without one. The black horizontal line at $y=50 h_{71}^{-1} \mathrm{kpc}$ corresponds to the criterion for determining whether or not a cluster has a CRS and the grey horizontal line at $y=12 h_{71}^{-1} \mathrm{kpc}$ corresponds to the worst uncertainty associated with the position of the $\mathrm{X}$-ray peak in the sample.

spectral analysis for these clusters for two reasons. First, since the main contribution to the synchrotron radio luminosity comes from the low-end of the radio frequency spectrum, this will result in precise determination of the synchrotron power in these systems. In our subsequent study, the energy in the radio emitting particles will be compared to the mechanical energy of the cavities, and thereby the partitioning of energy between radiation and mechanical (cavity) energy can be measured. Second, with dense enough spectral sampling, spectral breaks may also be visible (as have already been seen in a few CCRSs in the HIFLUGCS sample, see below). A spectral break in a system with cavities is an extremely useful observable since it is representative of the time since the last injection event or particle production, and therefore, a good indicator of the age of the cavity emission. The CCRSs, which we already know to show spectral breaks are presently being cross-checked with presence of cavities for a future study.

For sources with no observations or confirmed detection below $100 \mathrm{MHz}$, the low-end of their spectra were constrained using the $74 \mathrm{MHz} V L A$ Low-frequency Sky Survey (VLSS) with an average point-source detection limit of $0.5 \mathrm{Jy} /$ beam and a resolution of $80^{\prime \prime}$. Shown in the left panel of Fig. 2 are the spectra of all CCRSs. Shown in the right panel of Fig. 2 are the spectra of only a subset of CCRSs that show interesting features such as spectral breaks and turn-overs indicative of spectral aging and synchrotron self-absorption, respectively. Amongst the CCRSs shown in the right panel, we note there are also a few clusters which show spectral steepening at low-frequencies, which we believe is due to the superposition of different radio components with different spectral properties (due to varying sizes and distances from the central engine).

The details of the radio data used for this work, such as the various frequencies and the corresponding flux-densities used to estimate the radio luminosities of the cluster central radio sources, along with the references can be found in Table 1. The synchrotron radiation is assumed to have a powerlaw spectrum given by $S(v) \propto v^{-\alpha}$, where $S(v)$ is the flux density at frequency $v$. Thus, the integrated rest-frame radio luminosities, $L_{R}$, of the CCRSs were calculated by step-wise integration:

$$
\begin{aligned}
L_{i+1} & =4 \pi D_{l}^{2} S_{0} \int_{v_{i}}^{v_{i+1}}\left(v / v_{0}\right)^{-\alpha_{i+1, i}} \mathrm{~d} v \\
& =4 \pi D_{l}^{2} \frac{S_{0} v_{0}^{\alpha_{i+1, i}}}{\left(1-\alpha_{i+1, i}\right)}\left(v_{i+1}^{\left(1-\alpha_{i+1, i}\right)}-v_{i}^{\left(1-\alpha_{i+1, i}\right)}\right),
\end{aligned}
$$

where $S_{0}$ is the flux density of the radio source at either of the two rest-frame frequencies, $v_{i}$ or $v_{i+1}, L_{i+1}$ is the radio luminosity in the frequency range $\left[v_{i}, v_{i+1}\right], \alpha_{i+1, i}$ is the spectral index between $v_{i}$ and $v_{i+1}$, and $D_{l}$ is the luminosity distance. The total radio luminosity was calculated by extrapolating the spectral indices obtained at the lowest observed frequency to $10 \mathrm{MHz}$ and at the highest observed frequency to $15 \mathrm{GHz}$. Thus, $L_{\mathrm{tot}}=\sum L_{i+1}$. Of all the clusters with CCRSs, 27 have (reliable) data at more than two frequencies and 18 have data at two frequencies. The remaining three clusters (A576, A3158 and A3562) have data at only one frequency and we used $\alpha=1$, the average spectral index of the CCRSs in our sample, to calculate their total radio luminosity.

Even though the formal errorbars for $L_{R}$ were derived using the background root-mean-square in the maps, these do not take into account the uncertainty arising due to the lack of knowledge of the shape of the radio spectra down to the lowest frequencies, except for a handful of radio sources wellstudied at all radio-frequency bands (such as, Hydra-A (A0780), Centaurus (A3526), A1795, A2029, A2052, A2199, A2597 and A4059). Radio sources often show a spectral turn-over at lowfrequencies. This is attributed to synchrotron self-absorption which kicks in with increasing optical depth and is manifested by a rising spectrum with $\alpha=-2.5$. Two examples of spectral turnovers can be easily seen in the right panel of Fig. 2. Considering the possibility of other CCRSs showing similar turn-overs, we calculated a lower-limit on $L_{\mathrm{R}}$ based on the assumption that the spectra of the CCRSs turn over right below the lowest observed frequency. The difference between the lower limit derived in this manner and the integrated radio luminosities assuming the continual of the spectra beyond the lowest observed frequency provides a more realistic, albeit conservative, uncertainty on $L_{R}$. This is the reason for having highly asymmetric errorbars for $L_{\mathrm{R}}$, as can also be seen in many of the plots.

In other works (e.g. Burns 1990; Peres et al. 1998), very often the comparisons between the quantities representing the cooling flow strength in clusters and the radio power of the BCGs are based solely on the monochromatic radio luminosities, such as, the $1.4 \mathrm{GHz}$ luminosities derived from NVSS or FIRST or $5 \mathrm{GHz}$ luminosities derived from the Green Bank Survey. A useful exercise followed up with our data was to compare the integrated radio luminosities to $L_{1.4 \mathrm{GHz}}$, where the latter is given by

$L_{1.4 \mathrm{GHz}}=4 \pi D_{l}^{2} S_{1.4 \mathrm{GHz}}(1+z)^{\alpha_{1.4, i}-1}$,

where $\alpha_{1.4, i}$ is the spectral index between $1.4 \mathrm{GHz}$ and one of the two neighboring frequencies. Shown in Fig. 3 is the integrated radio luminosity versus the monochromatic radio luminosity at $1.4 \mathrm{GHz}$. Also shown is the best-fit powerlaw derived using the FITEXY least-squares line-fitting routine (Press et al. 1992) given by

$\frac{L_{\mathrm{R}}}{10^{42} h_{71}^{-2} \mathrm{ergs} \mathrm{s}^{-1}}=a \times\left(\frac{L_{1.4 \mathrm{GHz}}}{10^{32} h_{71}^{-2} \operatorname{ergs~s}^{-1} \mathrm{~Hz}^{-1}}\right)^{b}$,

where $a=1.04 \pm 0.03$ and $b=0.98 \pm 0.01$. This algorithm allows for fitting in only one parameter $\left(L_{\mathrm{R}}\right.$ in this case) but takes 

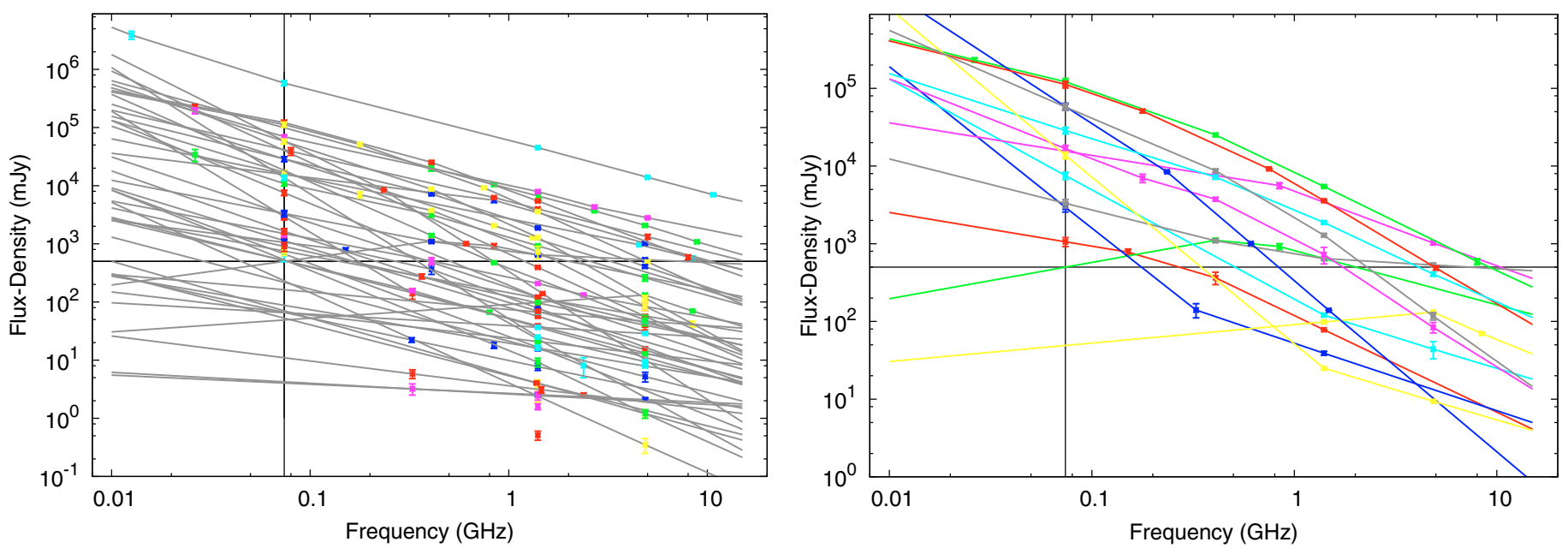

Fig. 2. The radio spectra of the cluster central radio sources. Shown in the left panel are the spectra of all 48 CCRSs, where the symbols are the actual measurements. Most of the measurements at $74 \mathrm{MHz}$ and $1.4 \mathrm{GHz}$ are taken from VLSS and NVSS, respectively. Shown in the right panel are the spectra of only those CCRSs which show spectral curvature. The black horizontal solid line represents the VLSS point-source sensitivity constraint at the VLSS observing frequency of $74 \mathrm{MHz}$ indicated by the black vertical line.

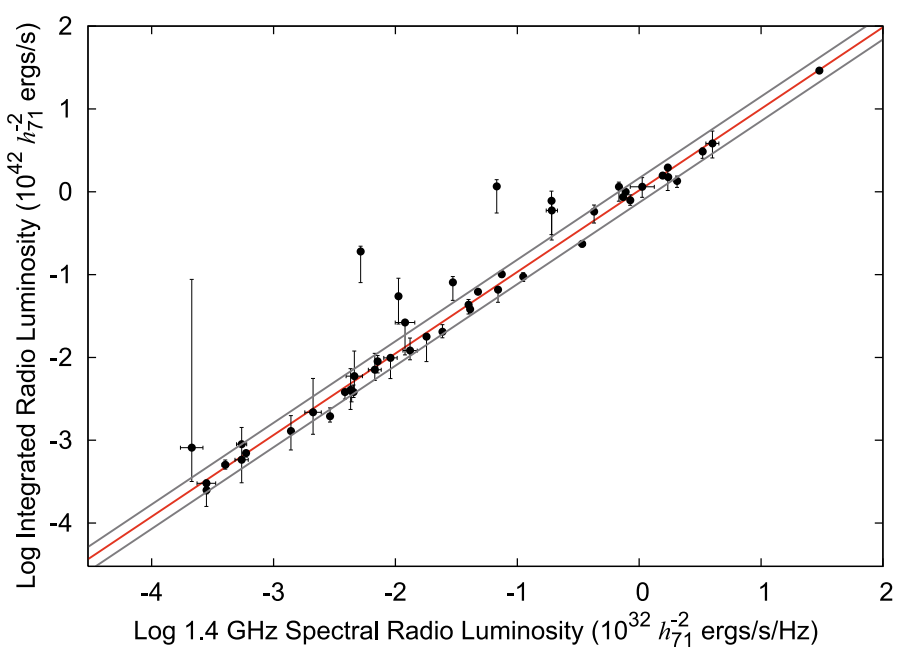

Fig. 3. A comparison between the radio luminosity integrated between $10 \mathrm{MHz}$ and $15 \mathrm{GHz}$ and the monochromatic radio luminosity at $1.4 \mathrm{GHz}$ (red solid line). The dotted grey lines correspond to $1-\sigma$ deviation above and below the best-fit line.

uncertainty in both $X$ and $Y$ into account. Our study shows that there is a fairly good correlation between the $L_{\mathrm{R}}$ and $L_{1.4} \mathrm{GHz}$. This is not surprising since once a pedestal value for radio sources is determined, the total power should scale with the spectral index, which for the CCRSs in our sample is quite similar and centers around unity. There are, however, a few CCRSs (such as 2A0335, A3376, MKW3S and A4038) which are inconsistent with the best-fit relation, all of which have spectralindices steeper than unity at low frequencies and were excluded while determining the best-fitting powerlaw. Hence, we conclude that even though the monochromatic luminosity (in this case at $1.4 \mathrm{GHz}$ ) is a good proxy for the total radio luminosity and may be used in cases where additional spectral information is not available, for precise radio correlations demanding least intrinsic scatter, the total radio power computed from detailed spectral analyses should be used.

\subsection{X-ray data}

The complete HIFLUGCS sample has Chandra observations and the data have been homogeneously reprocessed using CIAO 3.2.2 and CALDB 3.0. For a detailed description of the $\mathrm{X}$-ray data-reduction, and data- and error-analysis, the reader is referred to Hudson et al. (2008). In the following we briefly describe only those cluster X-ray parameters that are meaningful in the context of the present work.

\subsubsection{Cluster parameters}

We extracted the central density profiles by fitting either a single or double $\beta$-model to the surface-brightness profile annuli. Similarly, the central temperature profiles were derived by fitting spectra to annuli to an absorbed thermal model. From the temperature and density profiles, the central cooling time at $r=0.004 R_{500}\left(0.4 \% R_{500}\right), t_{\text {cool }}$, entropy, $K$, and cuspiness, $\alpha$, were calculated as per the following expressions:

$$
\begin{array}{r}
t_{\text {cool }}=\frac{5}{2} \frac{\left(n_{\mathrm{i} 0}+n_{\mathrm{e} 0}\right) k T_{48}}{n_{\mathrm{e}}^{2} \Lambda\left(T_{48}\right)} \quad ; \quad K(r)= \\
\alpha T(r) n_{\mathrm{e}}^{-2 / 3}(r) \\
\alpha=-\frac{\operatorname{dlog}\left(n_{\mathrm{e}}\right)}{\operatorname{dlog}(r)}
\end{array}
$$

where $n_{\mathrm{i} 0}$ and $n_{\mathrm{e} 0}$ are the central ion and electron densities, respectively, determined at $r=0.004 R_{500}, T_{48}$ is the average temperature of the $0-0.048 R_{500}$ region, $\Lambda\left(T_{48}\right)$ is the cooling function for a plasma at $T_{48}$ and $n_{\mathrm{e}}(r)$ is the electron density at a given radius $r$ from the cluster center. The cuspiness is calculated at a distance $r=0.04 R_{500}$ from the cluster center. Here, $R_{500}$ is the radius within which the average cluster mass density is 500 times higher than the critical density of the Universe.

To derive the cluster entropy profiles, the best fit density profiles were binned in steps of $2^{\prime \prime}$. Then for each bin, the value of temperature corresponding to that radius was adopted to calculate the entropy for that bin using the expression in Eq. (4). Since the annuli created to derive the temperature profiles need not necessarily coincide with the 2 " density bins, in the case where there was a jump in the temperature within a density bin, the average value of the two temperatures was used. 
The virial temperature of the cluster, used as a scaling parameter in some of the cross-correlations presented in the forthcoming sections, was determined by fitting the temperature profiles to broken powerlaws. This was done so as to prevent the cool-core gas from biasing the estimate for the global "viral" temperature, $T_{\text {vir. }}$. In those cases, where the inner powerlaw had a declining slope towards the center, which is representative of the cool gas at the centers of CC clusters, we excluded the core region as determined from the break in the powerlaw from the fit. From the estimate of $T_{\mathrm{vir}}$, the virial mass, $M_{500}$, within $R_{500}$ was determined using the relation by Finoguenov et al. (2001), $M_{500}=a k T_{\text {vir }}^{b} 10^{13} M_{\odot}$, where $a=2.5 \pm 0.2 \mathrm{~h}_{71}^{-1}$ and $b=1.676 \pm 0.054$.

The classical mass deposition rates, $\dot{M}_{\text {classical }}$, were derived from the gas temperature and density profiles. $\dot{M}_{\text {classical }}$ is the ratio of the total gas mass within the cooling radius, $r_{\text {cool }}$, defined as the region at which the $t_{\text {cool }}<7.7 \mathrm{Gyr}$, to the cooling time at this radius. Hence, $\dot{M}_{\text {classical }}(r)=M_{\text {gas }}\left(r<r_{\text {cool }}\right) / t_{\text {cool }}\left(r_{\text {cool }}\right)$ is a measure of the rate at which the mass should be dropping out of the X-ray band provided there is no source of heating. As the main focus for this work is on the central parts of clusters where cooling is most dominant, the X-ray analysis was performed only for the core regions.

\section{Results}

\subsection{CC and NCC cluster fractions: With and without a central radio source}

A well-known problem related to cooling-flows has been that of choosing an apt diagnostic for determining a cool-core cluster. To separate out the cool-core clusters from the non-cool-core ones, Hudson et al. (2008) searched for a bimodality in several of the X-ray observed and derived quantities, such as, the central cooling time, $t_{\text {cool }}$, the cooling radius defined as the radius out to which $t_{\text {cool }}<7.7 \mathrm{Gyr}$, the central entropy, the central density, the central luminosity, the mass deposition rate, the central temperature drop and the slope in surface brightness profile, wherein "central" refers to $0.004 R_{500}\left(0.4 \%\right.$ of $\left.R_{500}\right)$. Based on the K-Mean Method (KMM) algorithm (Ashman et al. 1994) as a test for bimodality (or tri-modality) in the parameters, Hudson et al. (2008) found $t_{\text {cool }}$ as the best measure for cooling to divide the CC and NCC clusters.

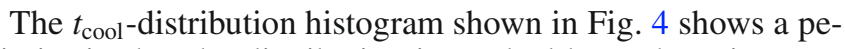
culiarity in that the distribution is marked by a sharp increase at $t_{\text {cool }}<1 \mathrm{Gyr}$; the fraction of clusters with $t_{\text {cool }}<1 \mathrm{Gyr}$ being $44 \%$. This oddity at $t_{\text {cool }}<1 \mathrm{Gyr}$ is also visible in two other properties, (1) the central temperature drop shown in the left panel of Fig. 5, defined as the ratio of the temperature in the innermost region to the virial temperature; and (2) the slope in the surface brightness profile, the "cuspiness", shown in the right panel of Fig. 5. Both the quantities show a break around a central cooling time of $t_{\text {cool }}<1 \mathrm{Gyr}$, even though the decrease in the central temperature drop is much more pronounced than the increase in the cuspiness. This result is also in concert with the recent findings of Rafferty et al. (2008), who investigated the relation between star formation, cooling activity in the ICM and AGN heating, based on a sample of 47 cluster center dominant galaxies (CDGs). According to their study, only the CDGs with cooling times below 0.8 Gyr exhibit positive color gradients, signifying an increase in star formation with decreasing distance from the galaxy center. The underlying reason for this behavior is not yet clear. It may be that the cool gas at the centers of galaxy clusters is feeding the star formation, in which case the

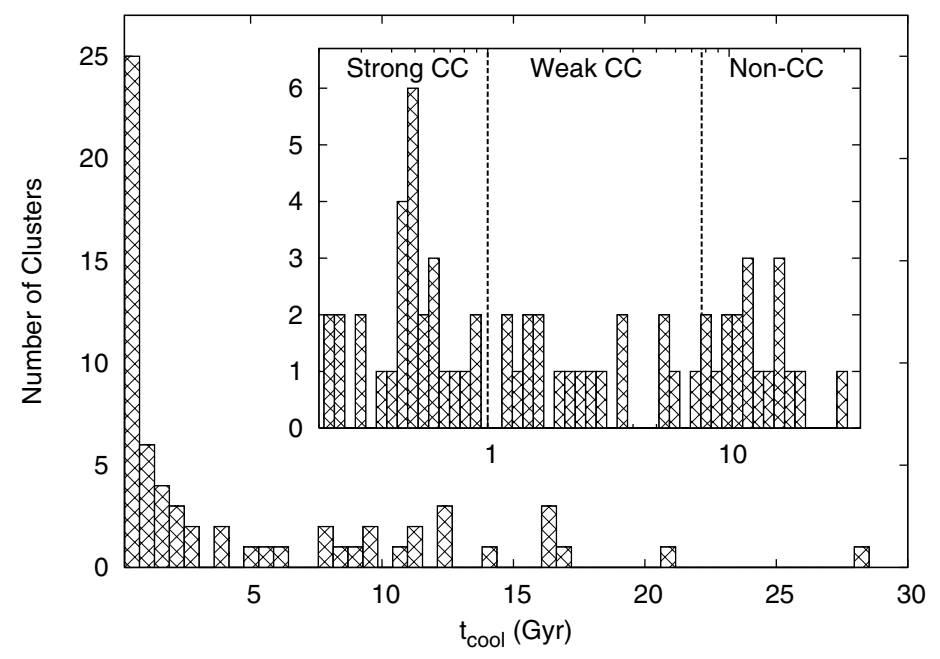

Fig. 4. The central cooling-time distribution clearly showing the steep rise in the fraction of clusters with cooling times shorter than 1 Gyr. For clarity, we show the distribution in both linear as well as log scale (inset panel) on the $x$-axis.

short cooling times should be tied with the time-scale over which the stars form and their light declines. It may well also be that AGN activity at the center of mass flows triggers star formation at the central regions. If the AGN feedback is responsible for regulating the cooling flows in clusters, then the cooling timescale may possibly reflect an intimate link with the time scale of the AGN outbursts (Sect. 4).

Based on the above results, we divided our sample into three categories, (1) strong cool-core (SCC) clusters with $t_{\text {cool }}<$ 1 Gyr, (2) weak cool-core (WCC) clusters with $1 \mathrm{Gyr}<t_{\text {cool }}<$ 7.7 Gyr (the upper limit of 7.7 Gyr is the usually assumed value for the cooling time corresponding to $z=1$, signifying the lookback time since the last major heating event, see McNamara \& Nulsen 2007) and (3) non-cool-core (NCC) clusters with $t_{\text {cool }}>7.7$ Gyr. These cuts result in $44 \%$ SCC clusters, $28 \%$ WCC clusters and $28 \%$ NCC clusters. The need to divide the distribution into three subgroups is bolstered by the fact that the KMM test showed adding a third sub-group improved the likelihood ratio, giving rise to a tri-modal distribution.

On cross-correlating the clusters with the presence of a CCRS, we find that all SCC clusters show cluster-center radio sources (Fig. 6). The resulting fraction of CCRSs amongst WCC clusters is $67 \%$ and that in NCC clusters is $45 \%$. A nonnegligible fraction of CCRSs in the WCC and NCC cluster population makes it uncertain whether there is a fundamental oneto-one correspondence between AGN heating and the lack of the expected cluster cooling. On the other hand, the probability of a BCG manifesting AGN activity clearly increases with decreasing cooling time. The next question that then arises is whether the radio luminosity of the central cluster radio source itself is correlated with $t_{\text {cool }}$. This is shown in Fig. 7 for SCC clusters and WCC clusters. From hereon, we refer to the combined set of SCC and WCC clusters as the cool-core (CC) clusters. This plot does not present a straight-forward interpretation of the interdependence between the AGN synchrotron power and the cooling time-scale. As a whole, there seems to be an anti-correlation between the two quantities but this seems to break down for clusters with $t_{\text {cool }} \lesssim 1$ Gyr. This apparent anti-correlation could be indicative of a need for more powerful AGN as heating agents in clusters with short $t_{\text {cool }}$. Yet the absence of any correlation between the $t_{\text {cool }}$ and $L_{\mathrm{R}}$ at short cooling times $(<\mathrm{Gyr})$ implies 

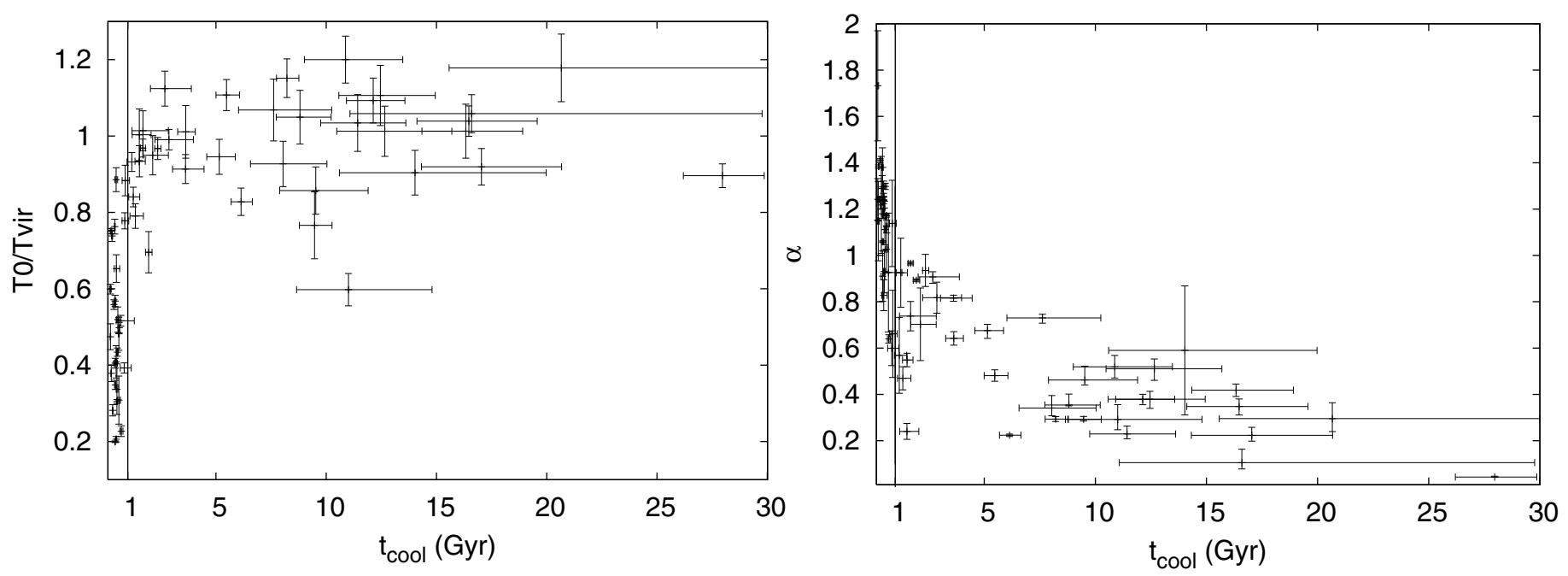

Fig. 5. Cooling time as an indicator of cool core. Left: the central temperature drop. Right: the slope in the surface brightness profile, cuspiness, at $4 \%$ of $R_{500}$.

that the AGN luminosity is more sensitive to a physical quantity other than the gas cooling time, such as possibly the mass deposition rate, $\dot{M}_{\text {classical }}$ (see Sect. 3.2).

Shown as crosses in Fig. 7 are four systems, NGC4646, NGC1550, NGC5044 and MKW4, which clearly depart from this trend and all of which are groups. A general property that the groups in our sample seem to share is that apart from having low temperatures (both virial and central), they also all have high central densities and subsequently short $t_{\text {cool }}$ (see Eq. (4)). On the other hand, the groups tend to have very steep density gradients resulting in small classical mass deposition rates, $\dot{M}_{\text {classical }}$. In other words, $\dot{M}_{\text {classical }}$ is more sensitive to the mass encompassed within the integration radius (see Sect. 2.2.1) than the cooling time at that radius [also see Fig. 6(G) of Hudson et al. 2008]. The behavior of $L_{\mathrm{R}}$ versus $\dot{M}_{\text {classical }}$ is investigated in Sect. 3.2. The fourth outlier, MKW4, is an interesting cluster under intensive study at radio wavelengths (see Sect. 3.3.1). Assuming the anti-correlation interpretation is correct, the best fit powerlaw excluding the four outliers derived using the bisector linear regression routine, BCES from Akritas \& Bershady (1996) is

$\frac{L_{\mathrm{R}}}{10^{42} h_{71}^{-2} \mathrm{ergs} \mathrm{s}^{-1}}=(0.041 \pm 0.016) \times\left(\frac{t_{\text {cool }}}{\mathrm{Gyr}}\right)^{-3.16 \pm 0.38}$.

This routine, like FITEXY, includes uncertainties in both the quantities but also additionally performs the minimization in both the dimensions. The Spearman rank correlation coefficient is -0.63 and the probability for the null-hypothesis is $8 \times 10^{-6}$.

For comparison with other works, we also determined the fraction of CCRSs in CC clusters, the fraction being $87 \%$. This is consistent with the result of Dunn \& Fabian (2006), who analyzed a low-redshift sample of clusters (B55) selected from pre-ROSAT data. Even though they find a slightly higher fraction $(95 \%)$ of CC clusters with CCRSs, they used a lower cut in $t_{\text {cool }}$ to determine CC clusters and, additionally, selected only those clusters which showed a central temperature drop $>2$. Using these criteria reduces the fraction of CC clusters in our sample to $25 \%$ but increases the fraction of CCRSs in CC clusters to $100 \%$. Similarly, Burns (1990) finds a somewhat lower fraction of $70 \%$ but the classification into CC and NCC clusters therein is based on the Hubble time. Using the Hubble time as the cut in $t_{\text {cool }}$ increases the fraction of CC clusters in our sample to $89 \%$ and reduces the fraction of CCRSs in CC clusters to $78 \%$.
We also bear in mind that the result by Burns (1990) is based on an incomplete sample and old X-ray Einstein data. Furthermore, the radio data used by Burns (1990) are based on monochromatic $5 \mathrm{GHz}$ VLA observations sensitive to largest structures of only about an arcminute, which in some cases might lead to overresolved structures and, hence, an under-estimation of the radio luminosity.

\subsection{Cooling and AGN activity}

We looked for correlations between the radio luminosity of the CCRSs and the X-ray-derived quantities to allow us to identify the underlying mechanisms that link the AGN activity and the cooling properties in clusters.

Shown in the left panel of Fig. 8 is the bolometric X-ray cluster luminosity in the energy range $0.01-40 \mathrm{keV}$ as inferred from ROSAT and ASCA measurements (Reiprich \& Böhringer 2002), $L_{\mathrm{X}}$, versus the integrated radio luminosity (see Sect. 2.1.2) for the 48 clusters with CCRSs. For the SCC clusters, shown as filled (blue) circles, there is a clear positive trend visible, although with a considerable spread. Since the X-ray luminosity is related through scaling relations to other global parameters of a cluster, such as the $T_{\text {vir }}$ and cluster mass, similar correlations may be obtained between the radio power of a CCRS and these quantities. This is the first time that the radio power of a centrally located AGN, the prime candidate for counteracting the cooling of the X-ray radiating ICM gas, has been shown to be correlated with the large-scale cluster properties. This result implies that there is a link between two regions, vastly differing in scales; the region over which AGN accretion takes place, which is no more than a few hundredth of a parsec, and the ICM, which extends out to 1-2 megaparsecs. Using the two-dimensional bisector linear regression routine $B C E S$, the trend between the $L_{\mathrm{R}}$ and $L_{\mathrm{X}}$ for SCC clusters may be quantified as below:

$\frac{L_{\mathrm{R}}}{10^{42} h_{71}^{-2} \mathrm{ergs} \mathrm{s}^{-1}}=a \times\left(\frac{L_{\mathrm{X}}}{10^{44} h_{71}^{-2} \mathrm{ergs} \mathrm{s}^{-1}}\right)^{b}$,

where $a=0.03 \pm 0.01$ and $b=1.38 \pm 0.16$. The Spearman rank correlation coefficient of the fit is 0.64 and the probability of their being no correlation is $2.7 \times 10^{-4}$. The Pearson correlation coefficient for the fit is larger and is equal to $\sim 0.76$. 


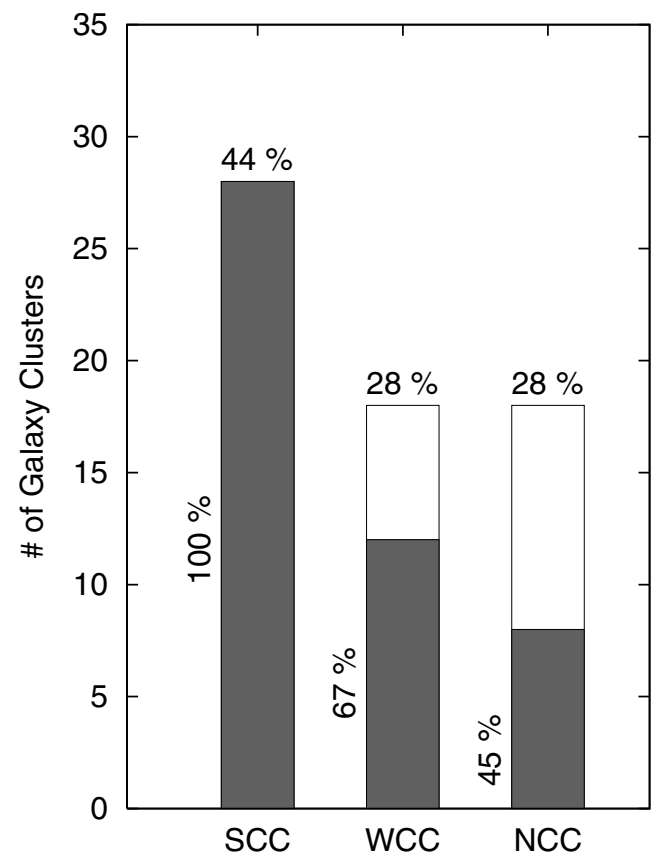

Fig. 6. The fraction of strong cool-core (SCC) clusters, weak coolcore (WCC) clusters and non-cool-core (NCC) clusters in the HIFLUGCS sample. Also shown are the fraction of clusters containing central radio sources for each category (shaded).

Note that luminosity-luminosity plots should be considered with caution due to the common redshift-dependence in both the quantities (Kembhavi et al. 1986; Akritas \& Siebert 1996; Merloni et al. 2006). Even though there are no censored data points (upper limits) for the category of SCC clusters, in that every SCC has radio source at the center, spurious correlation may still be introduced due to the common dependence on the distance. In order to check for such an occurrence, we simulated randomized radio and X-ray luminosities confined to the observed ranges following the distributions, $n\left(L_{\mathrm{X}}\right) \mathrm{d} L_{\mathrm{X}} \propto L_{\mathrm{X}}^{-0.7}$ (Böhringer et al. 2002) and $n\left(L_{\mathrm{R}}\right) \mathrm{d} L_{\mathrm{R}} \propto L_{\mathrm{R}}^{-0.78}$ (Nagar et al. $2005)$, where $n$ is the source number density. We assigned randomly distributed redshifts to the randomized luminosity data sets, according to the law $n \sim D_{l}^{3}$. These luminosities were reobserved after applying the X-ray flux limit, $f_{\mathrm{X}}(0.1-2.4) \mathrm{keV} \geq$ $2 \times 10^{-11} \mathrm{erg} \mathrm{s}^{-1} \mathrm{~cm}^{-2}$, the same as that for the HIFLUGCS sample, and the radio flux limit, $1.5 \mathrm{mJy}$ the average point-source detection limit for NVSS. The resulting Pearson correlation coefficients, $\rho_{\mathrm{P}}$, were compared to the observed one. Based on these simulations, we compute the probability of having $\rho_{\mathrm{P}}>0.76$ and the correlation slope equal to or greater than that observed for a completely randomized set of X-ray and radio luminosities as less than $1 \%$ (a spuriously induced correlation should produce a slope of around unity). This fraction increases to $\sim 2.5 \%$ if instead the Spearman correlation coefficient is used and, if in addition, the observed probability of null hypothesis is used as a further constraint, i.e., the probability of null hypothesis for the simulated data sets should be lower than $2.8 \times 10^{-4}$, then this fraction decreases to $\sim 1.5 \%$. Thus, we conclude that the probability of the observed correlation between the cluster X-ray luminosity and the radio luminosity of a CCRS to be spurious is very unlikely. However, that such an induced correlation is possible at a level of $\sim 3 \%$ in the worst case scenario, is worth keeping in mind for past and future studies on similar topics.

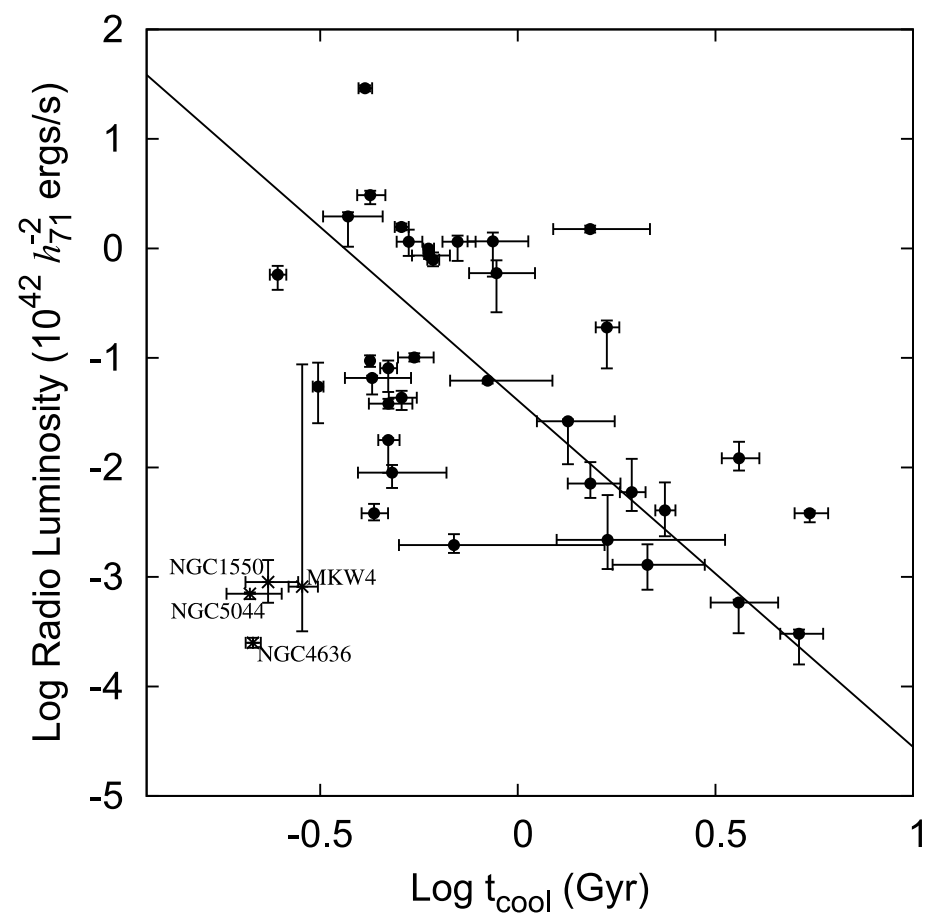

Fig. 7. The central cooling-time versus the integrated radio luminosity for the CCRSs in CC clusters (SCC + WCC). The black solid line represents the anti-correlation trend which breaks down for clusters with $t_{\text {cool }}<1$ Gyr. The labeled clusters are outliers with peculiar properties (see text for more).

Shown in the right panel of Fig. 8 is the radio luminosity versus $\dot{M}_{\text {classical }}$. This plot shows an even stronger trend than that seen with $L_{X}$. This further strengthens the likelihood of a coupling between gas cooling and the magnitude of the AGN activity. The NCC clusters do not appear on this plot since these by definition have no cooling radius, that is the central cooling time for these clusters is greater than $7.7 \mathrm{Gyr}$, implying zero mass deposition rates.

There are two interesting subsets of clusters pertaining to the right panel of Fig. 8, which deserve attention. The first subset comprises clusters which lack a CCRS but have $\dot{M}_{\text {classical }}>$ $1 M_{\odot} \mathrm{yr}^{-1}$, and the second subset, not shown in Fig. 8, comprises clusters which have a CCRS but for which $\dot{M}_{\text {classical }}=0$. The former subset (denoted by black triangles in Fig. 8) consists of: A1650, A2589, A2657 and A1060, with mass deposition rates $(93.7 \pm 28.2) M_{\odot} \mathrm{yr}^{-1},(19.9 \pm 12.3) M_{\odot} \mathrm{yr}^{-1}$ $(15.4 \pm 10.9) M_{\odot} \mathrm{yr}^{-1}$ and $(6.6 \pm 3.0) M_{\odot} \mathrm{yr}^{-1}$ respectively. In order to understand the behavior of the above four clusters, which are all WCC clusters, and to find out whether there exists a quantity that separates them from the other WCC clusters with a central radio source, we examined the entropy profiles of these clusters. Entropy is a powerful tool which provides information about two cluster parameters simultaneously - the temperature and the density; $K(r)=k T(r) n(r)^{-2 / 3}$, where $r$ is the radius from the cluster center. Shown in Fig. 9 are the entropy files of all but two WCC clusters. The two exceptions are A3266 and A3667, which have no CCRS but also have classical mass deposition rates consistent with zero. In other words, these are cases at the border line between WCC and NCC clusters and have, therefore, been excluded from Fig. 9. As can be seen, the entropy profiles of these four clusters (shown as color curves with symbols other than "+" symbols) are in no sense different from the rest. If anything, the profiles of A1650, A2589 and A1060 seem 

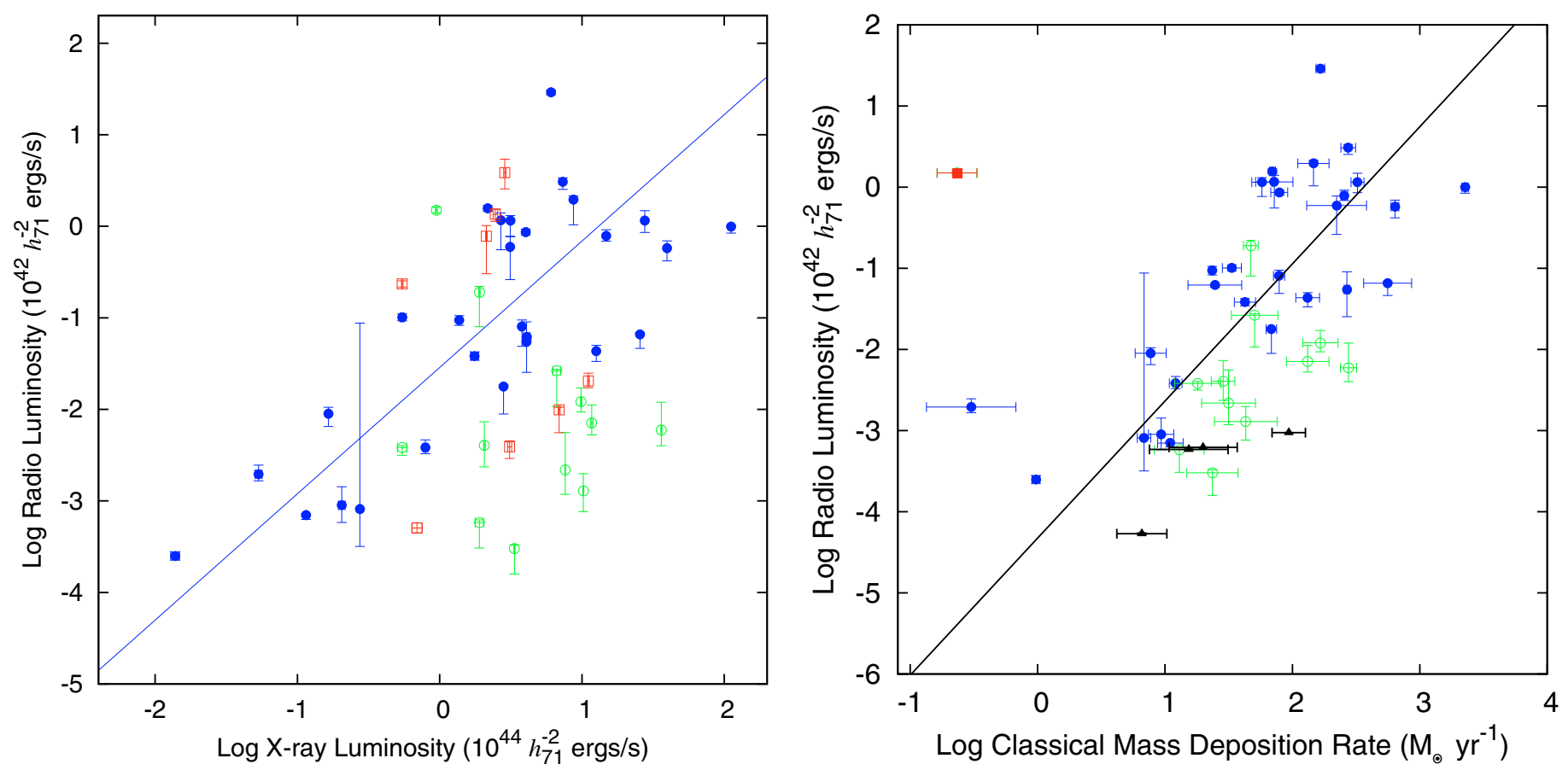

Fig. 8. Radio and X-ray correlation plots. Left: total radio luminosity vs. ROSAT bolometric X-ray luminosity for SCC (filled blue circles), WCC (open green circles) and NCC (open red squares) clusters. Right: total radio luminosity vs. the classical mass deposition rate for SCC clusters (filled blue circles) and WCC clusters (open green circles). The black triangles are four WCC clusters with $\dot{M}_{\text {classical }}>1$ and no central radio source. Hence, these are only upper limits. The filled red square on top left is A2634, also a WCC cluster. The solid black line is the best fit through CC clusters (SCC and WCC clusters combined) excluding A2634.

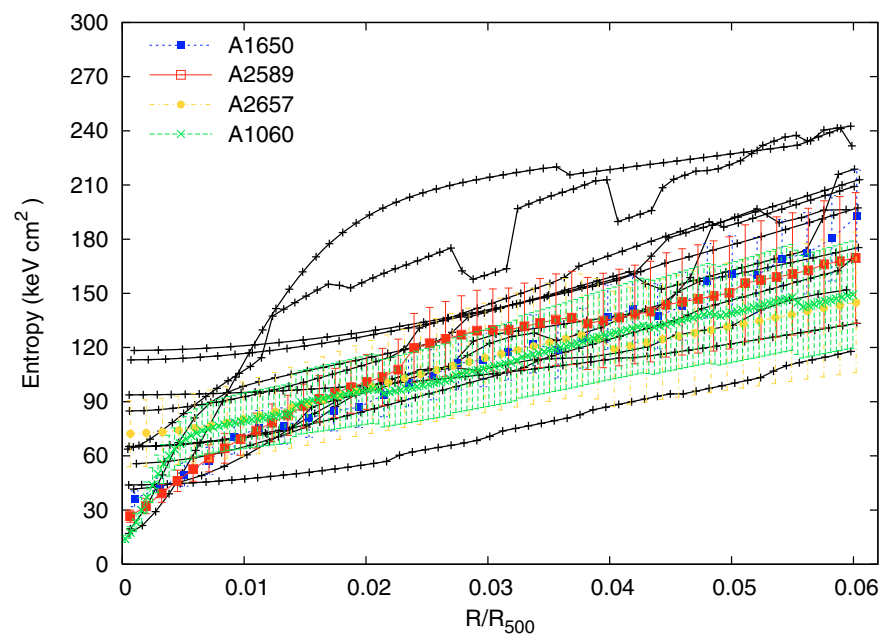

Fig. 9. The entropy profiles of the WCC clusters. All with a central radio source are shown in black (plus) symbols and four without are shown in colored (non-plus) symbols. The errorbars on those with a central radio source are omitted for clarity. The strong jumps apparent in some of the entropy profiles are insignificant relative to the errorbars.

to continue to fall with decreasing clustercentric distance. This implies a steady increase in the gas density with decreasing radius in these clusters and, hence, relatively strong cooling. What is the source of heating in these clusters?

Donahue et al. (2005) investigated one of the above radioquiet CC clusters, A1650, using Chandra observations and proffered one of the following two explanations for the absence of a radio AGN at the cluster center; (1) either the cluster has not reached the point where heating is necessary; or (2) the cluster experienced a major heating event about 1 Gyr ago such that it has not required feedback since then. Their conclusions are based on a lack of central temperature gradient in A1650 and a markedly raised central entropy as compared to other CC clusters with radio emission. Although the Chandra observations used by us (including $200 \mathrm{ks}$ that became publicly available in 2008) also imply an insignificant central temperature drop $\left(T_{0} / T_{\text {vir }} \sim 0.8\right)$, the estimated central entropy is not any higher than the average central entropy of the rest of the WCC clusters (Fig. 9).

Although, all of the above four clusters pose a serious threat to the AGN-regulated feedback fabric in cool-core clusters, A1650 is most intriguing due to a high value of discrepancy between the expected and measured mass deposition rates $\left[\dot{M}_{\text {classical }} \sim(93.7 \pm 28.2) M_{\odot} \mathrm{yr}^{-1}\right.$ and $\dot{M}_{\text {spec }}<0.7 M_{\odot} \mathrm{yr}^{-1}$, where $\dot{M}_{\text {spec }}{ }^{2}$ is the spectral mass deposition rate]. Interestingly, that there has been a mention of a weak detection of a radio source at the center of A1650 by Dunn \& Fabian (2006), which in turn is based on the VLA detection at $327 \mathrm{MHz}$ by Marković et al. (2004), who give the total flux-density of the radio source at this frequency as $59 \mathrm{mJy}$. But we have been unable to re-confirm this claim using the same observations as used by Marković et al. (2004) down to $3 \mathrm{mJy}$, three times the background noise. As also pointed out by Donahue et al. (2005), there is neither an indication of a past AGN outburst, either in the form of low-brightness diffuse lobe emission or cavities in the X-ray emission, nor are there any signatures of a recent merger. This cluster, along with its three companions, deserves further study in order to analyze other possible sources of heating such as conduction, intracluster supernovae or preheating.

The second subset corresponding to clusters with zero classical mass deposition rates but which contain a CCRS consists

\footnotetext{
${ }^{2}$ A detailed description of how $\dot{M}_{\text {spec }}$ is calculated can be found in Hudson et al. (2008).
} 
of: A3391, A3395s, A3376, A0400, A1656, A3158, A2147, MKW8 (in decreasing order of $L_{\mathrm{R}}$ ). These are NCC by definition and show signs of cluster mergers at different stages, as do most of the other NCC clusters in our sample (Hudson et al. 2008), based either on their X-ray properties (presence of subclumps or non-negligible separation between the X-ray emission peak and emission weighted centroid) or radio properties (presence of radio halos or relics or both). But contrary to the clusters in this subset, the remaining NCC clusters are devoid of a central radio source, supporting the idea that the presence of AGN is tightly correlated to gas cooling in clusters. The obvious question that then surfaces is, how does this subset fit into the AGNheating and gas-cooling machinery? There are several solutions to this apparent contradiction. Firstly, a well-known fact - AGN are found at all locations in clusters and not only at the cluster centers (although with an increasing probability with decreasing clustercentric distance, see Morrison \& Owen 2003; Best et al. 2007). There also exist field-AGN with no apparent reservoir of bulk cool gas, such as is available in clusters. Hence, there evidently are mechanisms other than those related to cluster cooling that can trigger radio nuclear activity in galaxies. Secondly, there is evidence that mergers may play a role in activating the central engines of AGN by transferring gas to the cluster galaxies and providing material for both, AGN accretion and also star formation (Owen et al. 1999). But alongside there also are contradictory findings according to which mergers may as well strip away gas from galaxies and result in inhibition of both the processes (e.g. see Giacintucci et al. 2004). Thirdly, a configuration containing a NCC cluster with zero mass deposition rate and a central radio source may be obtained through a merger between a NCC cluster and a CC cluster, latter harboring a central AGN, in such a way that it results in disruption of the cool core and leaves behind only traces of the past cooling activity. The simulations of Burns et al. (2008) indeed show that NCC clusters are formed as a result of major mergers right at the beginning of their evolution, whereby they grow in time at the expense of $\mathrm{CC}$ clusters. As an example, A2634 is a WCC cluster based on the central cooling time but in most other respects is closer to being a NCC cluster than a WCC cluster. According to Hudson et al. (2008), the X-ray morphology of this cluster is consistent with that of a merging cluster. Yet it has a cool core (short $\left.t_{\text {cool }}\right)$, and so A2634 might be a strong candidate for such a case where the cool core is being destroyed by a major merger.

Excluding the cluster on the upper-left corner (A2634) of Fig. 8 and the aforementioned subsets of outliers, the powerlaw fit for SCC and WCC clusters using the $B C E S$ routine gives,

$\frac{L_{\mathrm{R}}}{10^{42} h_{71}^{-2} \mathrm{ergs} \mathrm{s}^{-1}}=a \times\left(\frac{\dot{M}_{\text {classical }}}{M_{\odot} \mathrm{yr}^{-1}}\right)^{b}$,

where $a=(4.7 \pm 5.0) \times 10^{-5}$ and $b=1.69 \pm 0.25$. It can be argued that this exercise may be more appropriate if we use the spectrally determined mass deposition rate, $\dot{M}_{\text {spec }}$, as opposed to the classical one, $\dot{M}_{\text {classical }}$, as the former gives the actual observed rate at which the gas is cooling out and accreting onto the supermassive black hole. $\dot{M}_{\text {spec }}$ is, in fact, the fuel for the central AGN and should be correlated with the AGN output. On the other hand, the question that we are trying to address here is whether the AGN output can account for the difference between the classical (predicted) and spectral (observed) mass deposition rate and, thereby, provide a solution to the cooling flow puzzle. In addition, excluding clusters for which $\dot{M}_{\text {spec }}$ is consistent with zero, the classical mass deposition rates exceed the spectral mass deposition rates on average by a factor of 15 . Hence,
$\left(\dot{M}_{\text {classical }}-\dot{M}_{\text {spec }}\right) \sim \dot{M}_{\text {classical }}$ and the correlation between the radio luminosity and the two types of mass deposition rates is expected to be similar. We also point out that the spectral mass deposition rates estimated using the ACIS instrument on Chandra are, due to its low spectral resolving power, only moderately accurate. Therefore, any cross-correlation with the spectral mass deposition rates will have a large uncertainty making robust interpretations difficult.

Finally, we interpret the strong correlation seen between $\dot{M}_{\text {classical }}$ and $L_{\mathrm{R}}$ as supporting evidence for a feedback system in which the AGN activity is more enhanced in clusters with higher mass deposition rates, and the AGN in turn quenches the cooling of gas by heating the ambient medium. We note that a similar result was obtained by Peres et al. (1998) between $5 \mathrm{GHz}$ spectral radio luminosity and the mass deposition rate determined with ROSAT, although for a much smaller subset constituting only 15 galaxy clusters of the B55 sample (Edge et al. 1990).

\subsection{Brightest cluster galaxies (BCGs)}

The brightest cluster galaxies are unique in terms of their highluminosity and proximity to the centers of their host clusters. The BCGs are extremely interesting objects and have long been subjects of a wide range of studies. At one extreme, their formation and evolution is closely tied with the Mpc scale cluster environment in which they reside. At the other extreme in the hierarchy of structure formation, BCGs are just one level above the $\sim 10^{-4}$ pc scale supermassive black holes (SMBH). The BCG bulge properties, such as the optical bulge luminosity and stellar velocity dispersion, obey certain scaling relations that permit indirect estimation of the mass of SMBHs (e.g. Kormendy \& Richstone 1995; Ferrarese \& Merritt 2000).

In this section, we correlate the BCG magnitudes or, equivalently, the mass of the SMBHs with the AGN radio luminosity and the large-scale X-ray properties of galaxy clusters. The mass of the SMBH, $M_{\mathrm{BH}}$, is derived using the scaling relation between the near-infrared (NIR) bulge magnitude and the inferred $M_{\mathrm{BH}}$, as deduced by Marconi \& Hunt (2003). Further, we test whether there are any distinctions in the BCGs properties amongst the three different types of clusters (SCC, WCC and NCC clusters).

The BCG apparent magnitudes were taken from the Two Micron All-Sky Survey (2MASS Skrutskie et al. 2006) Extended Source Catalog $\left(\mathrm{XSC}^{3}\right)$. We used the 2MASS total magnitudes in $K$-band $(2.16 \mu \mathrm{m}), \mathrm{km}_{\mathrm{ext}}$, estimated from extrapolation of the surface brightness profiles (SBP). The extended source detection limit for $K$-band at $10-\sigma$ is 13.5 mag and the uncertainties range from $0.02-0.23 \mathrm{mag}$ with a mean of $0.06 \mathrm{mag}$. In short, the 2MASS SBPs have been derived from fitting a modified Sersic function to the elliptical radial light distribution of the BCGs. The total magnitudes are estimated from summing two terms. The first term corresponds to the isophotal magnitude estimated from fitting an ellipse to the standard isophote of mean surface brightness of $K_{20}=20 \mathrm{mag} \operatorname{arcsec}^{-2}$. The second term is derived by integrating the best-fitting Sersic law starting from the standard isophote, $r_{20}$, out to a delimiting isophote, which is typically about four scale lengths. This 2MASS strategy insures that the total flux of a galaxy is recovered.

The BCGs in 2MASS were located by searching around the brightest cluster galaxy in each cluster using an initial compilation, kindly provided by Heinz Andernach, based on the available data at NASA/IPAC Extragalactic Database

3 http://irsa.ipac.caltech.edu/Missions/2mass.html 
$\left(\mathrm{NED}^{4}\right)$ and Hyperleda ${ }^{5}$. All the BCGs were found within $5^{\prime \prime}$ of the given search position except in the case of three clusters, A2204 (SCC), A2065 (WCC) and A2163 (NCC), where, based on visual inspection, 2MASS did not manage to locate the right galaxies (i.e. even though within $5^{\prime \prime}$ of the given search position, the putative BCGs were not found in the 2MASS-XSC catalog). For these three clusters, we retrieved the 2MASS $K$-band Atlas images which have a plate scale of $1 " /$ pix, and fitted the BCG surface brightness distributions using the two-dimensional galaxy-fitting program, GALFIT (Peng et al. 2002). In order to be consistent with the 2MASS fitting routines, we restricted the functional form to Sersic models. While the SBPs of the BCGS of A2065 and A2163 were well-reproduced with a single-component Sersic models, the BCG in A2204 required a double-component Sersic model. For comparisons of the $K$-band magnitudes between the 2MASS-XSC and GALFIT estimates, we carried out tests by applying GALFIT to a few of the BCGs present in the XSC catalog and, hence, with known 2MASS magnitudes. We found that whereas GALFIT systematically underestimates the magnitude for bright BCGs $\left(\mathrm{km}_{\mathrm{ext}}<10 \mathrm{mag}\right)$ by about $10 \%$, the GALFIT magnitudes are consistent with the 2MASS-XSC magnitudes for faint BCGs $\left(\mathrm{km}_{\mathrm{ext}}>10 \mathrm{mag}\right)$ to within $3 \%$. Since the GALFIT magnitudes of the BCGs in A2204, A2065 and A2163 are all fainter than 12 mag (but brighter than the detection limit of $13.5 \mathrm{mag}$ ), we deem them to be trustworthy to within $5 \%$.

The apparent magnitudes from 2MASS were corrected for Galactic extinction using values from Schlegel et al. (1998), the typical correction values being small - on the order of $\sim 10^{-2}$, and were then converted into absolute magnitudes using the redshifts compiled from NED. We did not apply any $k$-correction since these galaxies are all nearby.

\subsubsection{Supermassive black hole mass and radio luminosity of the BCG}

There has been a lot of debate over the use of BCG scaling relations for determinations of $M_{\mathrm{BH}}$. This is due to the fact that many BCGs are often accompanied by low surface brightness envelopes extending out to, as far as, several hundred kiloparsecs (Gonzalez et al. 2005). These are the well-known "cD galaxies". The extended envelopes, also known as intracluster light (ICL) (Lin \& Mohr 2004), are thought to either represent debris accumulated over the merger history of the BCG or from tidal stripping of other cluster galaxies. The extended emission may as well originate from stars forming out of the condensed gas in cooling clusters. An important investigation we will follow-up in a subsequent paper is to study how the luminosity of these envelopes correlates with cooling parameters, such as the $t_{\text {cool }}$, $\dot{M}_{\text {classical }}, \dot{M}_{\text {spec }}$ etc. This requires careful decomposition of the BCG light profile into an inner component, associated just with the galaxy, and an outer flatter component representing the ICL. Seigar et al. (2007) fitted analytical models with two Sersic components to separately measure the profiles of the central and extended parts of $5 \mathrm{cD}$ galaxies and showed that the contribution of the envelopes to the total light is around $60 \%$ to $80 \%$.

Despite the above factors, recent studies (Batcheldor et al. 2007; Marconi \& Hunt 2003) have shown that the SMBH masses derived from the scaling relation using the NIR bulge magnitudes of the BCGs are consistent with those derived from

\footnotetext{
4 http://nedwww.ipac.caltech.edu

5 http://leda-univ-lyon $1 . f r$
}

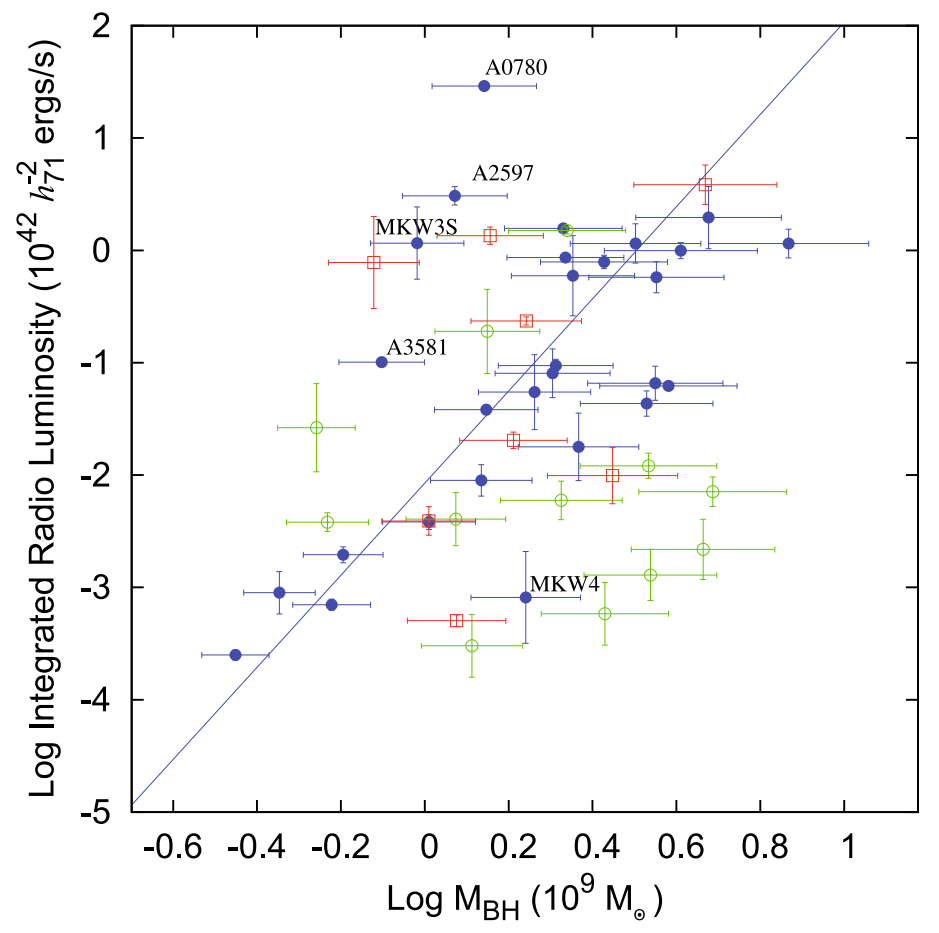

Fig. 10. The mass of the SMBH versus the total radio luminosity of the BCG in SCC (blue filled circles), WCC (green open circles) and NCC (red open squares) clusters. Visible is a weak trend given by solid blue line for the SCC clusters such that the $L_{R}$ of the BCG increases with the SMBH mass. Labeled are the five SCC outliers.

stellar velocity dispersions $\left(\sigma_{\mathrm{v}}\right)$, both the relations yielding similar amount of scatter. This is not quite true for $V$ - or $B$-band magnitudes though, where the scatter is much higher compared to the preferred $\sigma_{\mathrm{v}}-M_{\mathrm{BH}}$ relation. Batcheldor et al. (2007) attribute the scatter to inclusion of luminosity from the outerenvelopes of the $\mathrm{cD}$ galaxies, which may be more pronounced in the $V$ - or $B$-band, especially if the outer-envelope light represents on-going star-formation, and which might not have anything to do with the central galaxy dynamics (however, see Lauer et al. 2007, for a difference of opinion). Based on the studies on the NIR bulge magnitude- $M_{\mathrm{BH}}$ relation, we use the following scaling relation (Marconi \& Hunt 2003) to derive the SMBH masses:

$\log _{10}\left(\frac{M_{\mathrm{BH}}}{M_{\odot}}\right)=a+b\left[\log _{10}\left(\frac{L_{\mathrm{BCG}}}{L_{\odot}}\right)-10.9\right]$,

where $a=8.21 \pm 0.07$ and $b=1.13 \pm 0.12$. In order to convert the absolute magnitudes into luminosities in units of $K$-band solar luminosity, we used the absolute $K$-band solar magnitude equal to 3.32 mag (Colina \& Bohlin 1997). In Fig. 10 we present the SMBH masses versus the $L_{\mathrm{R}}$ for the 48 CCRSs (BCGs) in our sample. Whereas on the whole there appears to be a poor correlation of increasing AGN radio output with increasing $M_{\mathrm{BH}}$, categorization of clusters based on $t_{\text {cool }}$ results in a trend to appear between the two quantities but only for the SCC clusters. The best-fit powerlaw using the two-dimensional fitting algorithm $B C E S$ for the SCC clusters is:

$\frac{L_{\mathrm{R}}}{10^{42} h_{71}^{-2} \mathrm{ergs} \mathrm{s}^{-1}}=(0.008 \pm 0.004) \times\left(\frac{M_{\mathrm{BH}}}{10^{9} M_{\odot}}\right)^{4.10 \pm 0.42}$.

The Spearman rank correlation coefficient of the fit is 0.46 and the probability of their being no correlation is $1.3 \times 10^{-2}$. 
The Pearson correlation analysis yields a larger correlation coefficient (0.59) and a much smaller probability of the nullhypothesis $\left(9 \times 10^{-4}\right)$.

Several studies have explored the correlation between the SMBH mass and radio luminosity of the embedded AGN, leading to contradictory results. Whereas it is clear that the radio loudness, the ratio of the radio to the optical luminosity of an AGN, is a strong function of the black hole mass (Laor 2000), whether or not there exists a tight relation between $L_{\mathrm{R}}$ and $M_{\mathrm{BH}}$ is still a matter of debate. Franceschini et al. (1998) derived a tight relation between the radio luminosity at $5 \mathrm{GHz}, L_{5} \mathrm{GHz}$, and $M_{\mathrm{BH}}$ using a sample of 13 nearby early-type weakly active galaxies, such that $L_{5} \mathrm{GHz} \propto M_{\mathrm{BH}}^{2.66}$. Lacy et al. (2001) also find a similar correlation for a sample of steep-spectrum radio sources, albeit leading to a flatter slope of 1.4. Although Laor (2000) confirmed this trend of increasing radio luminosity with increasing black hole mass using a much larger sample of 29 nearby galaxies and 89 PG (Palomar-Green) quasars, the trend they obtain is weak and presents a lot of scatter. As an example of dissenting views, Liu et al. (2006) find no relation of $L_{5} \mathrm{GHz}$ against the black hole mass. On the other hand, they find a strong correlation between the jet power and the black hole mass, where they used the flux density of the radio lobes at $151 \mathrm{MHz}$ as a proxy to determine the jet power. Based on the above results it is not yet clear how the radio luminosity of an AGN scales with the black hole mass. It may be that different black hole efficiencies, conversion rates from the total AGN power to the radiative power of the jet and large-scale environmental effects from source to source, cause the radio luminosity to display a wide range of values for a given black hole mass.

In pursuit of determining whether or not there is an underlying physical mechanism that ties the radio power to the mass of the SMBH, Fig. 10 presents an interesting outlook. We investigate for the first time the dependence of $L_{\mathrm{R}}$ on $M_{\mathrm{BH}}$ taking into consideration the different environments (SCC, WCC and NCC clusters) in which the centrally located radio sources reside. According to the simulations of Burns et al. (2008), CC clusters grow an enhanced cool-core gradually and steadily via small mergers, unlike NCC clusters which experience major mergers early in their history. Hence, repetitive small mergers in addition to providing material for the growth of the cool core in $\mathrm{CC}$ clusters, may also result in a quasi-steady mass accretion rate onto the SMBH in CC clusters. The fact that only the CCRSs in SCC clusters show some level of correlation between $M_{\mathrm{BH}}$ and $L_{\mathrm{R}}$ implies that the AGN output in these systems is proportional to the average mass-accretion rate onto the black hole, thereby, balancing the radiative losses.

At the time of writing this article, MKW4, an outlier below the best-fit line in Fig. 10, had no low-frequency $(<1.4 \mathrm{GHz})$ radio data available. This inspired us to acquire $327 \mathrm{MHz} V L A$ data for this cluster, which is work-in-progress. MKW4 is an interesting cluster in that high-frequency $(1.4 \mathrm{GHz}$ and $5 \mathrm{GHz}) V L A$ archival data showed a cluster of closely spaced point-sources $\gtrsim 1$ arcmin to the NE of the BCG radio emission. Furthermore, radio emission from the $\mathrm{BCG}$ was only detected at $5 \mathrm{GHz}$ and not at $1.4 \mathrm{GHz}$, the latter having had only 2-min on-source integration time. The preliminary $327 \mathrm{MHz}$ image of MKW4 revealed the same feature NE of the BCG but due to insufficient resolution, it still remained unclear whether this emission was associated with the nucleus of the CCRS or corresponded to a high-redshift cluster system; the latter conclusion being based on the proximity of these point sources to each other. Assuming that the emission in the NE reflected the region of intense interaction between the lobe and ICM and, hence, was a part of the central radio source, would have caused the $L_{\mathrm{R}}$ to increase 30 fold and for it to no longer be an outlier. To confirm this hypothesis, we acquired dynamic VLA time at $1.4 \mathrm{GHz}$ in $\mathrm{CnD}$ configuration with $3 \mathrm{~h}$ integration time. Even though the new $1.4 \mathrm{GHz}$ data show no signature of a connection between the CCRS and the bunch point-source emission in the NE, it clearly shows emission from the CCRS, which went undetected with the old archival data. This itself increased the total radio output by more than an order of magnitude bringing this cluster closer towards the observed $M_{\mathrm{BH}}-L_{\mathrm{R}}$ trend for the SCC clusters (this change is incorporated in Fig. 10). The follow-up study of the CCRS in MKW4 underlines the importance of obtaining reliable spectra of radio sources.

In addition to MKW4, there are four other outliers in Fig. 10 which lie above the best-fit relation: A0780, A2597, MKW3S and A3581. We believe these systems might be ones which have experienced powerful radio outbursts in recent past resulting in the present AGN heating rate to be greater than the average rate at which the mass has been accreting onto the $\mathrm{SMBH}$ since the formation of the cool core. Similarly, MKW4 may reflect a CCRS that has been caught just at the beginning of another heating cycle and has yet to reach its peak radio activity. These outliers, both below and above the best-fit line may be reflective of the episodic nature of CCRSs in some systems. Another plausible reason for these outliers may be the weakness in the underlying assumption of the integrated luminosity of the BCG as a robust indicator of the bulge luminosity in all the cases.

\subsubsection{Large-scale cluster properties and BCGs}

Inherent scalings between BCGs and clusters have been implied in numerous observational studies (e.g. Lin \& Mohr 2004; Brough et al. 2008) as well as cosmological simulations (Zheng et al. 2007; Cooray \& Milosavljević 2005). In this context, formation and evolution of BCGs and its dependence on the host cluster is an important tool to understand these scalings observed between the BCG luminosity and the host halo mass and X-ray luminosity. There are several proposed BCG evolution scenarios to support these observations, such as (1) dynamical-friction governed galactic cannibalism; (2) rapid mergers between galaxies during the epoch of cluster formation; (3) co-evolution of BCGs with cluster growth due to mergers embracing the paradigm of hierarchical structure formation; and (4) coolingflows. While the first two scenarios may contribute significantly during the early epoch of BCG formation, Lin \& Mohr (2004) and Brough et al. (2008) argue that the BCGs co-evolve with the host clusters via mergers with the BCGs of the falling subclusters, which lead to subsequent growth of the BCG luminosity with increasing cluster mass.

We present in this section the BCG-host cluster correlations for the HIFLUGCS sample, with the aim to extend previous studies by analysing a low-z flux-limited sample unique in its completeness and homogeneity in the ways of obtaining the X-ray and NIR quantities. The following study is unique in an additional aspect in that we bear in mind the possibility of different growth histories for the BCGs corresponding to CC and NCC clusters.

Shown in Fig. 11 is $M_{500}$ versus $L_{\mathrm{BCG}}$ in the left panel and $L_{\mathrm{X}}$ versus $L_{\mathrm{BCG}}$ in the right panel. Shown in different symbols and colors are the three different types of clusters. Whereas both the panels clearly indicate that the BCG grows with the cluster size, the left panel additionally shows a segregation between the SCC clusters (blue filled circles) and the non-SCC clusters. A possible reason for such a separation may be related to the continuous 

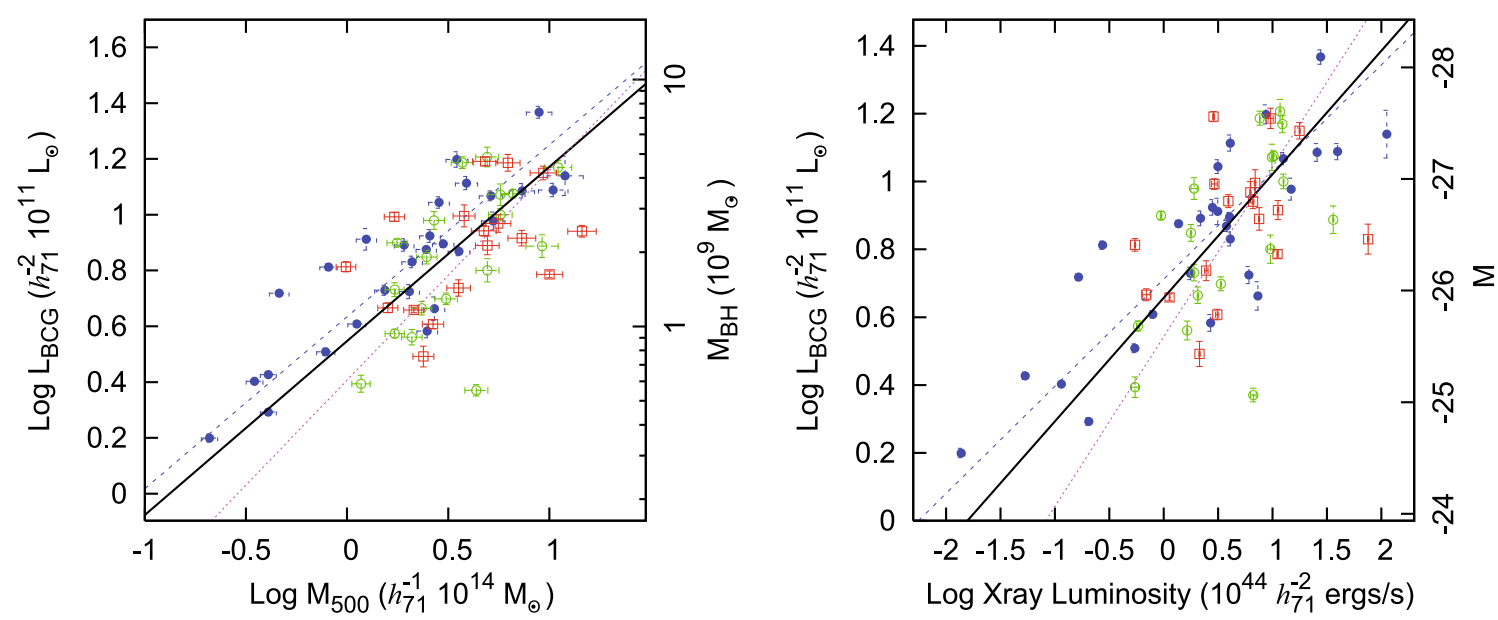

Fig. 11. The 2MASS $K$-band total magnitudes of the 64 BCGs in the HIFLUGCS sample versus $M_{500}$ in the left panel and ROSAT bolometric cluster X-ray luminosity in the right panel. The different symbols and colors denote SCC (filled blue circles) clusters, WCC (non-filled green circles) clusters and NCC (non-filled red squares) clusters. The solid (black), the dashed (blue) and the dotted (pink) lines denote the best-fit powerlaws for all clusters, only SCC clusters, and only non-SCC (WCCs+NCCs) respectively.

growth of BCGs, as already mentioned in Sect. 3.3.1, due to iterative small-scale mergers through which the CC clusters grow. The BCGs in NCCs, on the other hand, form constituents of nonrelaxed cluster environments with a history of one or more major mergers, and the subsequent heating at the central regions may hinder further BCG growth at the same rate as that of BCGs in $\mathrm{CC}$ clusters. This argument is supported by the fact that on comparing the radial profiles of numerically simulated CC and NCC clusters, Burns et al. (2008) observe an excess of baryons in CC clusters relative to NCC clusters.

An interesting note is that this segregation appears only between $L_{\mathrm{BCG}}$ versus $M_{500}$ and not between $L_{\mathrm{BCG}}$ versus $L_{\mathrm{X}}$. However, a similar intrinsic separation for CC and NCC clusters is seen between $T_{\text {vir }}$ and $L_{\mathrm{X}}$. This is attributed to the fact that at a given temperature, SCC clusters have a higher luminosity as compared to non-SCC clusters due to an increased gas density at the center. This causes the SCC clusters to form an envelope towards the higher luminosity end. Since $M_{500}$ has been derived from $T_{\text {vir }}$, it may be that the resulting magnitude of separation between $M_{500}$ and $L_{\mathrm{X}}$ cancels with that between $L_{\mathrm{BCG}}$ and $M_{500}$.

The estimated best-fit powerlaw for the $L_{\mathrm{BCG}}-M_{500}$ relation based on the BCES algorithm is:

$$
\frac{L_{\mathrm{BCG}}}{10^{11} h_{71}^{-2} L_{\odot}}=a \times\left(\frac{M_{500}}{10^{14} M_{\odot} \mathrm{yr}^{-1}}\right)^{b}
$$

where $a=3.525 \pm 0.277$ and $b=0.624 \pm 0.054$ for all clusters, $a=4.305 \pm 0.290$ and $b=0.616 \pm 0.005$ for the SCC clusters only and $a=2.552 \pm 0.362$ and $b=0.752 \pm 0.095$ for the non-SCC clusters (CC clusters) only. The Spearman rank correlation coefficients for the fits are $0.67,0.87$ and 0.62 for all, SCC and non-SCC clusters, respectively. Looking at the fit results for all clusters and SCC clusters only, it is clearly seen that the SCC clusters have a higher normalization by about $20 \%$ (since the two fits have the same slope within the 1- $\sigma$ errorbars, the normalizations may be directly compared). The slope of the obtained $L_{\mathrm{BCG}}-M_{500}$ relation is steeper than the values derived in other works, which tend to center around 0.3 , even though presenting a wide range from 0.1 to 0.5 (Lin \& Mohr 2004; Brough et al. 2008; Popesso et al. 2007; Hansen et al. 2007).
Going a step further, we may combine Eqs. (8) and (10) to derive a relation between the SMBH mass and the cluster mass:

$$
\frac{M_{\mathrm{BH}}}{10^{9} M_{\odot}}=a \times\left(\frac{M_{500}}{10^{14} M_{\odot} \mathrm{yr}^{-1}}\right)^{b}
$$

where $a=0.98 \pm 0.08$ and $b=0.61 \pm 0.06$ for all clusters, $a=1.15 \pm 0.09$ and $b=0.63 \pm 0.06$ for the SCC clusters only and $a=0.75 \pm 0.14$ and $b=0.70 \pm 0.12$ for the non-SCC clusters. Even though indirectly derived using the BCG bulge luminosities, such a correlation could be indicative of a fundamental relation between the host cluster halo and central SMBH similar to the galaxy bulge mass-black hole mass relation.

Similarly, the best-fit powerlaw for the $L_{\mathrm{BCG}}-L_{\mathrm{X}}$ relation is

$\frac{L_{\mathrm{BCG}}}{10^{11} h_{71}^{-2} L_{\odot}}=a \times\left(\frac{L_{\mathrm{X}}}{10^{44} h_{71}^{-2} \text { ergs s}^{-1}}\right)^{b}$

where $a=4.54 \pm 0.34$ and $b=0.36 \pm 0.03$ for all clusters, $a=5.15 \pm 0.38$ and $b=0.32 \pm 0.03$ for the SCC clusters only and $a=3.49 \pm 5.09$ and $b=0.50 \pm 0.07$ for the non-SCC clusters. The Spearman rank correlation coefficients for the fits are $0.68,0.82$ and 0.55 for all, SCC and the non-SCC clusters, respectively. The $L_{\mathrm{BCG}}-L_{\mathrm{X}}$ and $L_{\mathrm{BCG}}-M_{500}$ relations show that there is a statistically significant difference in slopes between SCC and nonSCC clusters. Similar correlations were obtained by Katayama et al. (2003) using a larger sample, which also contains the HIFLUGCS sample. However, their results show much higher scatter between the BCG luminosity-host halo mass, which is due likely to the use of optical $B$-band magnitudes which have larger errorbars of mean value $\sim 0.2$ mag. Further, Katayama et al. (2003) correlated the optical magnitude versus the total mass of the host cluster defined as the integrated mass within a fixed metric radius of $5 \mathrm{Mpc}$, for which both, statistical as well as systematic uncertainties are expected to be larger.

The above results, which highlight a strong dependence of the BCG NIR magnitudes on the scale of the host cluster, however, are at variance with those obtained by Brough et al. (2002). They claim that any division between BCGs in low- $L_{X}$ and high$L_{X}$ clusters as seen in high- $z$ clusters, disappears for clusters with $z \leq 0.1$. The input low- $z$ sample $(z \leq 0.1)$ studied by Brough et al. (2002) consisted of 150 Abell clusters with a flux limit of 
$f_{\mathrm{X}}(0.1-2.4) \mathrm{keV}=3 \times 10^{-12} \mathrm{erg} \mathrm{s}^{-1} \mathrm{~cm}^{-2}$ in the ROSAT hard band $(0.5-2.0 \mathrm{keV})$. After matching these with the 2MASS catalog resulted in a final sample comprising 76 clusters with only those BCGs which have robust 2MASS magnitude measurements in the $K$-band. We argue that the contradiction in results might stem from differences in the aperture radii used, within which the magnitudes are calculated. The 2MASS database provides galaxy magnitudes based on a suite of apertures. While Brough et al. (2002) employ integrated magnitudes measured using circular apertures of a fixed metric radius of $12.5 h_{71}^{-1} \mathrm{kpc}$, we use, as explained above, the total aperture radii by extrapolating the SBPs, the mean of which for the BCGs in our study is $\sim 50 h_{71}^{-1} \mathrm{kpc}$. From this we conclude that the total BCG magnitude is a better quantity to use, as opposed to the BCG core magnitude, for detecting correlations with global cluster properties.

\section{Discussion}

The riddle of cooling-flows in clusters has continued to baffle us. Recent high-resolution Chandra images revealed radio-loud AGN embedded in the centers of cool-core (CC) clusters surrounded by regions emptied of the X-ray emitting gas, suggesting a strong tie between the cluster central radio source and the cooling of ICM. Since the discovery of numerous AGN-blown bubbles in the atmospheres of CC clusters, various modes of energy transfer from the AGN to the ICM have been investigated. While the most successful mode has turned out to be dissipation of energy stored in the radio bubbles as they rise buoyantly through the ICM, previous studies have fallen short of a thorough investigation of the relation between the radio luminosity of the centrally located AGN and cooling properties of a $\mathrm{CC}$ cluster.

It is now a widely accepted fact that the AGN activity is triggered by gas accretion onto the central black hole. Playing devil's advocate, it may then be argued that the AGN output is only to be expected to scale with the mass accretion rate. Under the assumption that the cool gas flows from the outer cluster regions to the very centers of the BCGs and serves as the fuel for the black hole, it is not surprising that the radio luminosity of an AGN should scale with the cluster mass, and also, even though to a lesser degree, the inverse of the cooling time of the gas. This explains the underlying trend seen in Fig. 8 between the $\dot{M}_{\text {classical }}$ and the radio output of CCRSs in CC clusters. However, the picture thus developed so far does not contain any ingredients reflecting on a self-regulated cycle formed between gas cooling, star formation and AGN heating. In other words, it may well be that even though AGN activity enhances with cluster scale, the cooling of ICM is regulated by an altogether different process, such as cluster mergers.

The first strong argument in favor of AGN-regulated heating comes from the observation that the AGN fraction increases with decreasing central cooling time, $t_{\text {cool }}$ being the best diagnostic to distinguish CC from NCC clusters. The study by Rafferty et al. (2008) shows that the central star-formation rate also is a strong function of $t_{\text {cool }}$ (see Sect. 3.1). That only clusters with short $t_{\text {cool }}<1$ Gyr have increasing on-going star formation with decreasing clustercentric distance, implies a chain of intricately linked processes which maintain heating and cooling rates in cluster atmospheres. These results together call for a feedback process in which AGN heating becomes more of a requisite in clusters with shorter cooling times. This may either be in form of huge AGN outbursts which heat the surrounding cluster gas, the effect of which lasts for several cooling cycles (such as Hydra-A,
MKW3S, A2597 and A3581), or in form of short-lived AGN outbursts which are repeated after short intervals. A recent study by Shabala et al. (2008) has shown that the CCRSs in more massive clusters undergo AGN outbursts more frequently than the AGN in their less massive counterparts. Additionally, radio source models employed by Shabala et al. (2008) show that the duration of the on-state of an AGN has the same relation with the stellar mass as the mass deposition rate has with the stellar mass (Best et al. 2005), suggesting the switching on and off of an AGN resulting directly from either availability or depletion of cool cluster gas.

More recently, Voit et al. (2008) have provided evidence that the AGN activity, ICM cooling and star-formation might all be linked together through the process of electron thermal conduction. According to their study, the efficacy of thermal conduction depends on the size of the temperature inhomogeneities relative to the critical length scale associated with conduction, $\lambda_{\mathrm{f}}$. The state of equilibrium between radiation losses and conduction gain can be equivalently expressed in terms of $\lambda_{\mathrm{f}}$ and $K$. Based on above arguments, conduction sets an entropy threshold such that only those clusters whose central entropy is less than $30 \mathrm{keV} \mathrm{cm}^{-2}$ show star formation, in the form of $\mathrm{H} \alpha$, and enhanced AGN activity. Above this threshold, conduction is a viable heating mechanism. Further, since gas entropy is very closely linked to the central cooling time through the relation, $t_{\text {cool }} \propto K^{3 / 2} / T$, such that $0.6 \mathrm{Gyr}(1 \mathrm{Gyr})$ corresponds approximately to $30 \mathrm{keV} \mathrm{cm}^{-2}\left(43 \mathrm{keV} \mathrm{cm}^{-2}\right)$, these observations are also in concordance with our results on the central temperature drop and cuspiness displayed in Fig. 5. The central entropy (or central cooling time) threshold may be an explanation for observing an abrupt central temperature drop and an increase in cuspiness for clusters with cooling times shorter than 1 Gyr. In the cluster regime with $t_{\text {cool }}<1 \mathrm{Gyr}$, AGN heating is the dominant balancing mechanism to cooling.

We point out that there is non-negligible scatter in Figs. 8 and 10, the origin of which could be either extrinsic or intrinsic. In the presence of an AGN-regulated feedback, an intrinsic scatter may imply that the synchrotron luminosity, which is only a small fraction of the total AGN output, is not a very reliable quantity to use to establish the AGN-ICM interaction. It has been noted in previous studies, that the ratio of kinetic to radiative AGN power indeed shows a broad range, from a few to a several thousands (e.g. Birzan et al. 2008; Bîrzan et al. 2004). Kinetic AGN luminosity may be a more robust measure of the total AGN feedback. For this, one requires the radio morphology of jets and lobes overlaid on X-ray images to help find or confirm the X-ray cavities. The census of X-ray cavities is highly incomplete since they are of very low contrast, yet they are important contributions to the heating budget. An extrinsic scatter in the plots would point at inaccurate measurements of observable parameters at both the wavebands, radio (incomplete spectral information) as well as X-ray (imprecise mass deposition rates due to spectral resolution power of ACIS on Chandra).

However, strong correlations found in this work between the total AGN radio power and various cluster parameters lend confidence in synchrotron luminosity as a fairly good measure of the cooling activity in clusters. These correlations also provide us with motivation to continue our work to acquire lowfrequency radio measurements for CCRSs which have no reliable data below $500 \mathrm{MHz}$ (constituting $35 \%$ of the HIFLUGCS sample, see Sect. 2.1.2). To achieve this goal, we are awaiting proprietary data for all but two of the remaining $35 \%$ clusters with VLA at $325 \mathrm{GHz}$ and with GMRT at dual-frequency band $610 \mathrm{MHz} / 235 \mathrm{MHz}$. 


\section{Conclusions}

We have presented a detailed joint analysis of the brightest complete local sample of galaxy clusters, HIFLUGCS, using highresolution X-ray data acquired from Chandra and radio data compiled from various sources spanning a wide range of frequencies. This study was conducted so as to explore the role of AGN in the centers of galaxy clusters accompanied with the cooling flow problem. The main results of this study are:

1. We find that the integrated radio luminosity $\left(L_{\mathrm{R}}\right)$ of a cluster central radio source is tightly correlated to its $1.4 \mathrm{GHz}$ monochromatic luminosity (see Fig. 3) with the exception of a few outliers. This correlation is quantified as $L_{\mathrm{R}} \propto$ $L_{1.4 \mathrm{GHz}}^{0.98 \pm 0.01}$ (Eq. (2)). To estimate the integrated radio luminosity of a CCRS, a special effort was made to compile lowfrequency radio measurements in order to get an accurate measure of the total radiative output of the centrally located AGN.

2. The best property to diagnose a cool-core cluster with high quality data is the central cooling time, $t_{\text {cool }}$. Based on $t_{\text {cool }}$, there is an increasing probability for the brightest cluster galaxy (BCG) closest to the X-ray peak emission to harbor an AGN with decreasing cooling time (Fig. 6). The percentage of AGN in three bins ordered in cooling time, strong cool core (SCC, $t_{\text {cool }} \leq 1$ Gyr), weak cool-core (WCC, 1 Gyr $\left.\leq t_{\text {cool }} \leq 7.7 \mathrm{Gyr}\right)$ and non-cool-core $\left(\mathrm{NCC}, t_{\text {cool }} \geq 7.7 \mathrm{Gyr}\right)$ clusters, is $100 \%, 67 \%$ and $45 \%$ respectively.

3. There is a trend between the $L_{\mathrm{R}}$ and $t_{\text {cool }}$ such that the former increases with decreasing cooling time. This is shown in Fig. 7, although with a large scatter especially towards short $t_{\text {cool }}$ where the trend appears to break down.

4. The total radio output of a CCRS scales with the cluster size (e.g. X-ray luminosity). This correlation is particularly noticeable in SCC clusters (see Fig. 8, left panel). The bestfit powerlaw for the SCC clusters is $L_{\mathrm{R}} \propto L_{\mathrm{X}}^{1.38 \pm 0.16}$ (Eq. (6)).

5. The total radio output of cool-core clusters (SCC and WCC clusters) shows a tight correlation with the classical mass deposition rate, $\dot{M}_{\text {classical }}$ (Fig. 8, right panel). The correlation is given by $L_{\mathrm{R}} \propto \dot{M}_{\text {classical }}^{1.69 \pm 0.25}$ (Eq. (7))

6. The radio luminosity of the central radio source shows a weak trend with the mass of the supermassive black hole, but this trend is seen only for the SCC clusters (Fig. 10). This trend is approximately given by $L_{\mathrm{R}} \propto M_{\mathrm{BH}}^{4.10 \pm 0.42}$.

7. The NIR bulge luminosity of the BCG (closest to the X-ray peak emission) shows a correlation at an unprecedented level with the global cluster properties, such as X-ray mass shown in the left panel of Fig. 11, and luminosity shown in the right panel of Fig. 11 (Eqs. (10) and 12).

While we have provided strong evidence of there being an abundance of CCRSs with enhanced radio luminosities in clusters where cooling activity is at its full thrust (SCC clusters), a feedback heating-cooling loop may require involvement of additional physical processes, such as conduction as mentioned above, to halt the cooling in WCC clusters.

Acknowledgements. The authors want to thank Heinz Andernach for providing information on the initial BCG search positions for 2MASS and Paul Nulsen, Tod Lauer and Douglas Richstone for helpful discussions and the internal referee, Manuel Perucho, for a beneficial feedback. The authors thank the external referee for a very interesting report and for provoking us to think about certain important issues in detail. R.M. acknowledges support from the Deutsche Forschungsgemeinschaft (DFG) through the Schwerpunkt Program 1177 (RE 1462/4). T.H.R and D.S.H. acknowledge support from the DFG through the Emmy Noether research grant RE 1462/2. Basic research in radio astronomy at the NRL is supported by 6.1 Base funding. This publication makes use of data products from the Two Micron All Sky Survey, which is a joint project of the University of Massachusetts and the Infrared Processing and Analysis Center/California Institute of Technology, funded by the National Aeronautics and Space Administration and the National Science Foundation. This research has made use of the NASA/IPAC Extragalactic Database (NED) which is operated by the Jet Propulsion Laboratory, California Institute of Technology, under contract with the National Aeronautics and Space Administration. We acknowledge the usage of the HyperLeda database (http://leda.univ-lyon1.fr).

\section{References}

Akritas, M. G., \& Bershady, M. A. 1996, ApJ, 470, 706

Akritas, M. G., \& Siebert, J. 1996, MNRAS, 278, 919

Allen, S. W. 1995, MNRAS, 276, 947

Ashman, K. M., Bird, C. M., \& Zepf, S. E. 1994, AJ, 108, 2348

Bagchi, J., \& Kapahi, V. K. 1994, J. A\&A, 15, 275

Batcheldor, D., Marconi, A., Merritt, D., \& Axon, D. J. 2007, ApJ, 663, L85

Benson, A. J., Bower, R. G., Frenk, C. S., et al. 2003, ApJ, 599, 38

Best, P. N., Kauffmann, G., Heckman, T. M., et al. 2005, MNRAS, 362, 25

Best, P. N., von der Linden, A., Kauffmann, G., Heckman, T. M., \& Kaiser, C. R. 2007, MNRAS, 379, 894

Binney, J., \& Tabor, G. 1995, MNRAS, 276, 663

Bîrzan, L., Rafferty, D. A., McNamara, B. R., Wise, M. W., \& Nulsen, P. E. J. 2004, ApJ, 607, 800

Birzan, L., McNamara, B. R., Nulsen, P. E. J., Carilli, C. L., \& Wise, M. W. 2008, ArXiv e-prints, 806

Blanton, E. L., Sarazin, C. L., McNamara, B. R., \& Wise, M. W. 2001, ApJ, 558, L15

Blanton, E. L., Sarazin, C. L., McNamara, B. R., \& Clarke, T. E. 2004, ApJ, 612, 817

Bock, D. C.-J., Large, M. I., \& Sadler, E. M. 1999, AJ, 117, 1578

Boehringer, H., Voges, W., Fabian, A. C., Edge, A. C., \& Neumann, D. M. 1993, MNRAS, 264, L25

Böhringer, H., Collins, C. A., Guzzo, L., et al. 2002, ApJ, 566, 93

Bower, R. G., Benson, A. J., Malbon, R., et al. 2006, MNRAS, 370, 645

Brough, S., Collins, C. A., Burke, D. J., Mann, R. G., \& Lynam, P. D. 2002 , MNRAS, 329, L53

Brough, S., Couch, W. J., Collins, C. A., et al. 2008, MNRAS, 385, L103

Burns, J. O. 1990, AJ, 99, 14

Burns, J. O., Hallman, E. J., Gantner, B., Motl, P. M., \& Norman, M. L. 2008, ApJ, 675, 1125

Chandran, B. D. G., \& Rasera, Y. 2007, ApJ, 671, 1413

Churazov, E., Sunyaev, R., Forman, W., \& Böhringer, H. 2002, MNRAS, 332, 729

Clarke, T. E., Blanton, E. L., \& Sarazin, C. L. 2004, ApJ, 616, 178

Cohen, A. S., Lane, W. M., Cotton, W. D., et al. 2007, AJ, 134, 1245

Colina, L., \& Bohlin, R. 1997, AJ, 113, 1138

Condon, J. J., Cotton, W. D., Greisen, E. W., et al. 1998, AJ, 115, 1693

Cooray, A., \& Milosavljević, M. 2005, ApJ, 627, L85

Croton, D. J., Springel, V., White, S. D. M., et al. 2006, MNRAS, 365, 11

Donahue, M., Voit, G. M., O’Dea, C. P., Baum, S. A., \& Sparks, W. B. 2005, ApJ, 630, L13

Dunn, R. J. H., \& Fabian, A. C. 2006, MNRAS, 373, 959

Edge, A. C., \& Frayer, D. T. 2003, ApJ, 594, L13

Edge, A. C., Stewart, G. C., Fabian, A. C., \& Arnaud, K. A. 1990, MNRAS, 245, 559

Edwards, L. O. V., Hudson, M. J., Balogh, M. L., \& Smith, R. J. 2007, MNRAS, 379,100

Fabian, A. C. 1994, ARA\&A, 32, 277

Fabian, A. C., Sanders, J. S., Allen, S. W., et al. 2003, MNRAS, 344, L43

Ferrarese, L., \& Merritt, D. 2000, ApJ, 539, L9

Finoguenov, A., Reiprich, T. H., \& Böhringer, H. 2001, A\&A, 368, 749

Franceschini, A., Vercellone, S., \& Fabian, A. C. 1998, MNRAS, 297, 817

Gentile, G., Rodríguez, C., Taylor, G. B., et al. 2007, ApJ, 659, 225

Giacintucci, S., Venturi, T., Bardelli, S., Dallacasa, D., \& Zucca, E. 2004, A\&A, 419, 71

Giacintucci, S., Venturi, T., Murgia, M., et al. 2007, A\&A, 476, 99

Gonzalez, A. H., Zabludoff, A. I., \& Zaritsky, D. 2005, ApJ, 618, 195

Guo, F., \& Oh, S. P. 2008, MNRAS, 384, 251

Hansen, S. M., Sheldon, E. S., Wechsler, R. H., \& Koester, B. P. 2007, ArXiv e-prints, 710

Heinz, S., Choi, Y.-Y., Reynolds, C. S., \& Begelman, M. C. 2002, ApJ, 569, L79 Hudson, D. S. et al. 2008, A\&A, submitted

Jones, C. Forman, W., Vikhlinin, A., et al. 2002, ApJ, 567, L115

Kaastra, J. S., den Boggende, A. J., Brinkman, A. C., et al. 2001, in X-ray Astronomy 2000, ed. R. Giacconi, S. Serio, \& L. Stella, ASP Conf. Ser., 234, 351 
Katayama, H., Hayashida, K., Takahara, F., \& Fujita, Y. 2003, ApJ, 585, 687 Kembhavi, A., Feigelson, E. D., \& Singh, K. P. 1986, MNRAS, 220, 51 Kormendy, J., \& Richstone, D. 1995, ARA\&A, 33, 581

Lacy, M., Laurent-Muehleisen, S. A., Ridgway, S. E., Becker, R. H., \& White, R. L. 2001, ApJ, 551, L17

Laor, A. 2000, ApJ, 543, L111

Lauer, T. R., Faber, S. M., Richstone, D., et al. 2007, ApJ, 662, 808

Lin, Y.-T., \& Mohr, J. J. 2004, ApJ, 617, 879

Liu, Y., Jiang, D. R., \& Gu, M. F. 2006, ApJ, 637, 669

Marconi, A., \& Hunt, L. K. 2003, ApJ, 589, L21

Marković, T. Owen, F. N., \& Eilek, J. A. 2004, in The Riddle of Cooling Flows in Galaxies and Clusters of galaxies, 61

Mathews, W. G., \& Brighenti, F. 2003, ARA\&A, 41, 191

Mathews, W. G., Faltenbacher, A., \& Brighenti, F. 2006, ApJ, 638, 659

McCarthy, I. G., Babul, A., Bower, R. G., \& Balogh, M. L. 2007, ArXiv e-prints, 706

McNamara, B. R. \& Nulsen, P. E. J. 2007, ARA\&A, 45, 117

McNamara, B. R., \& O'Connell, R. W. 1989, AJ, 98, 2018

McNamara, B. R., Wise, M., Nulsen, P. E. J., et al. 2000, ApJ, 534, L135

McNamara, B. R., Wise, M. W., Nulsen, P. E. J., et al. 2001, ApJ, 562, L149

Merloni, A., Körding, E., Heinz, S., et al. 2006, New Astron., 11, 567

Miller, N. A. 2005, AJ, 130, 2541

Mittaz, J. P. D., Kaastra, J. S., Tamura, T., et al. 2001, A\&A, 365, L93

Morrison, G. E., \& Owen, F. N. 2003, AJ, 125, 506

Nagar, N. M., Falcke, H., \& Wilson, A. S. 2005, A\&A, 435, 521

Nulsen, P. E. J., et al. 2006, ArXiv Astrophysics e-prints

O’Hara, T. B., Mohr, J. J., Bialek, J. J., et al. 2006, ApJ, 639, 64

Owen, F. N., Ledlow, M. J., Keel, W. C., \& Morrison, G. E. 1999, AJ, 118, 633

Patnaik, A. R., \& Singh, K. P. 1988, MNRAS, 234, 847

Peng, C. Y., Ho, L. C., Impey, C. D., \& Rix, H.-W. 2002, AJ, 124, 266

Peres, C. B., Fabian, A. C., Edge, A. C., et al. 1998, MNRAS, 298, 416

Peterson, J. R., Paerels, F. B. S., Kaastra, J. S., et al. 2001, A\&A, 365, L104

Peterson, J. R., Kahn, S. M., Paerels, F. B. S., et al. 2003, ApJ, 590, 207

Pope, E. C. D., Pavlovski, G., Kaiser, C. R., \& Fangohr, H. 2005, MNRAS, 364, 13
Popesso, P., Biviano, A., Böhringer, H., \& Romaniello, M. 2007, A\&A, 464, 451 Press, W. H., Teukolsky, S. A., Vetterling, W. T., \& Flannery, B. P. 1992, Numerical recipes in FORTRAN. The art of scientific computing (Cambridge: University Press), 2nd ed

Rafferty, D., McNamara, B., \& Nulsen, P. 2008, ArXiv e-prints, 802

Rafferty, D. A., McNamara, B. R., Nulsen, P. E. J., \& Wise, M. W. 2006, ApJ, 652,216

Reiprich, T. H., \& Böhringer, H. 2002, ApJ, 567, 716

Ricker, P. M., \& Sarazin, C. L. 2001, ApJ, 561, 621

Roychowdhury, S., Ruszkowski, M., Nath, B. B., \& Begelman, M. C. 2004, ApJ, 615,681

Ruszkowski, M., Brüggen, M., \& Begelman, M. C. 2004, ApJ, 615, 675

Sakelliou, I., Peterson, J. R., Tamura, T., et al. 2002, A\&A, 391, 903

Sanders, J. S., Fabian, A. C., Allen, S. W., et al. 2008, MNRAS, 385, 1186

Scannapieco, E., Silk, J., \& Bouwens, R. 2005, ApJ, 635, L13

Schlegel, D. J., Finkbeiner, D. P., \& Davis, M. 1998, ApJ, 500, 525

Seigar, M. S., Graham, A. W., \& Jerjen, H. 2007, MNRAS, 378, 1575

Shabala, S. S., Ash, S. A., Alexander, P., \& Riley, J. M. 2008, ArXiv e-prints

Sijacki, D., Springel, V., di Matteo, T., \& Hernquist, L. 2007, MNRAS, 380, 877

Silk, J., \& Rees, M. J. 1998, A\&A, 331, L1

Skrutskie, M. F., Cutri, R. M., Stiening, R., et al. 2006, AJ, 131, 1163

Slee, O. B., Roy, A. L., Murgia, M., Andernach, H., \& Ehle, M. 2001, AJ, 122, 1172

Sun, M., Jones, C., Murray, S. S., et al. 2003, ApJ, 587, 619

Tamura, T., Kaastra, J. S., Peterson, J. R., et al. 2001, A\&A, 365, L87

Valentijn, E. A., \& Bijleveld, W. 1983, A\&A, 125, 223

Venturi, T., Bardelli, S., Zagaria, M., Prandoni, I., \& Morganti, R. 2002, A\&A, 385,39

Voigt, L. M., \& Fabian, A. C. 2004, MNRAS, 347, 1130

Voit, G. M., \& Donahue, M. 2005, ApJ, 634, 955

Voit, G. M., Cavagnolo, K. W., Donahue, M., et al. 2008, ArXiv e-prints, 806

von der Linden, A., Best, P. N., Kauffmann, G., \& White, S. D. M. 2007, MNRAS, 379, 867

Xu, H., Kahn, S. M., Peterson, J. R., et al. 2002, ApJ, 579, 600

Zheng, Z., Coil, A. L., \& Zehavi, I. 2007, ApJ, 667, 760 


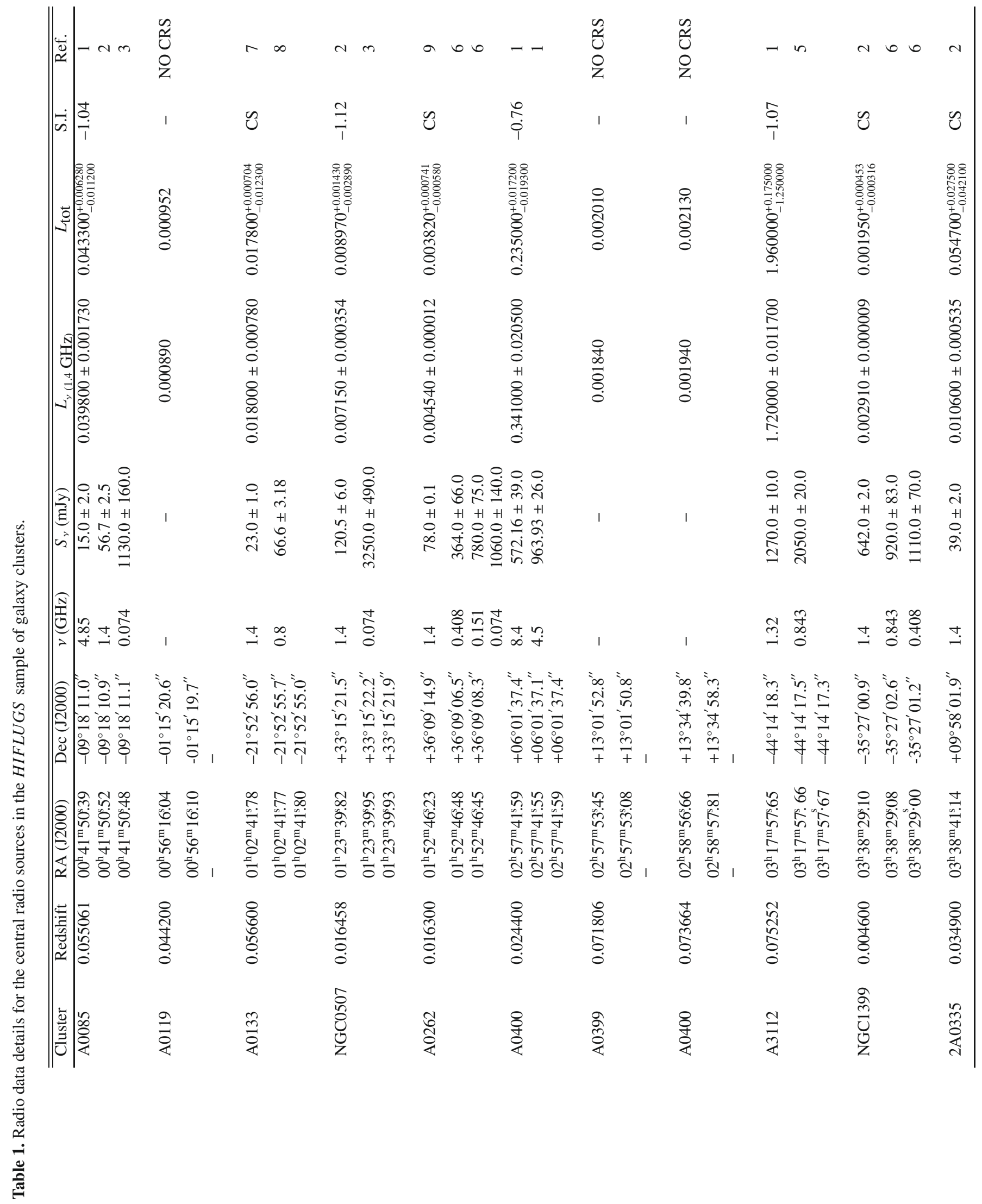


R. Mittal et al.: AGN heating in the HIFLUGCS sample of galaxy clusters, Online Material p 2

\begin{tabular}{|c|c|c|c|c|c|c|c|c|c|c|}
\hline $\mid \ddot{\mathbb{e}}$ & $\subseteq m-N$ & in & $N-$ & $0 \mathrm{Nm}$ & $-N m$ & $\begin{array}{l}\tilde{N} \\
\text { đै } \\
0 \\
z\end{array}$ & $0 \mathrm{Nm}$ & - & 000606 & 06 \\
\hline 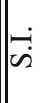 & के & $\stackrel{\circ}{i}$ & $\begin{array}{l}\infty \\
\grave{\varphi}\end{array}$ & $\stackrel{\tilde{m}}{T}$ & $\stackrel{8}{\circ}$ & I & U & 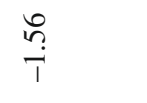 & $\stackrel{n}{i}$ & U \\
\hline 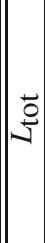 & 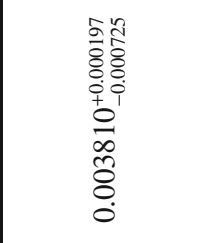 & $\begin{array}{l}\text { to. } \\
\text { o. } \\
\text { o. } \\
0.0 \\
+0 \\
0 \\
0 \\
0 \\
0 \\
0 \\
0 \\
0 \\
0\end{array}$ & $\begin{array}{l}8 \\
8 \\
0.00 \\
0.00 \\
0.0 \\
\vdots \\
0 \\
0 \\
0 \\
0 \\
0 \\
0\end{array}$ & 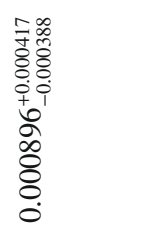 & 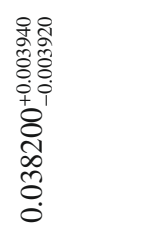 & $\begin{array}{l}0 \\
\stackrel{\infty}{0} \\
\stackrel{0}{0} \\
0\end{array}$ & 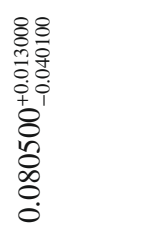 & 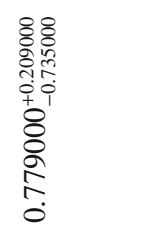 & 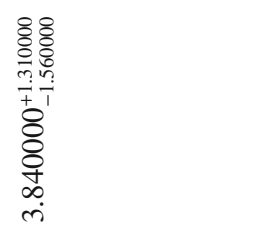 & 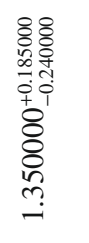 \\
\hline 息 & $\begin{array}{l}\infty \\
\infty \\
\infty \\
0 \\
0 \\
0 \\
+1 \\
0 \\
0 \\
\infty \\
0 \\
0 \\
0 \\
0\end{array}$ & $\begin{array}{l}\stackrel{8}{0} \\
\overline{0} \\
0 \\
0 \\
+1 \\
0 \\
0 \\
\bar{\sigma} \\
\dot{0} \\
\dot{0}\end{array}$ & $\begin{array}{l}8 \\
8 \\
0 \\
0 \\
0 \\
0 \\
+1 \\
8 \\
8 \\
0 \\
0 \\
0 \\
0\end{array}$ & 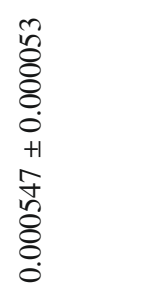 & $\begin{array}{l}\frac{9}{0} \\
\frac{1}{0} \\
8 \\
0 \\
0 \\
+1 \\
8 \\
0 \\
\\
0 \\
0\end{array}$ & $\frac{8}{\stackrel{8}{+}}$ & 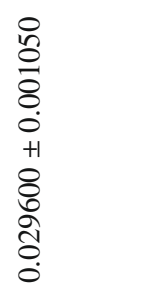 & $\begin{array}{l}8 \\
8 \\
8 \\
0 \\
0 \\
+1 \\
0 \\
8 \\
0 \\
0 \\
0 \\
0\end{array}$ & 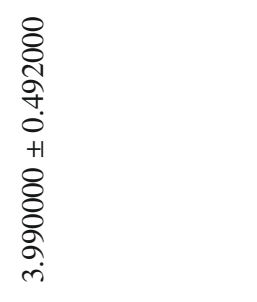 & 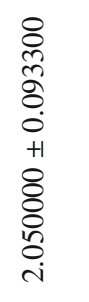 \\
\hline$\tilde{E}$ & 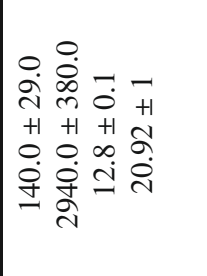 & $\begin{array}{l}\tilde{m} \\
i \\
+1 \\
\tilde{n} \\
\infty\end{array}$ & 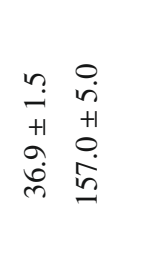 & 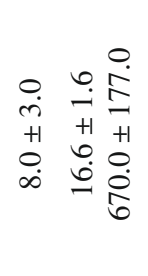 & 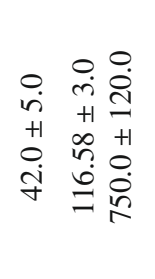 & I & 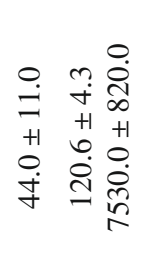 & 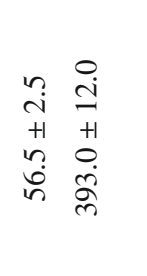 & 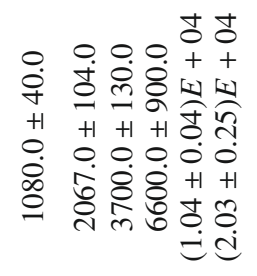 & 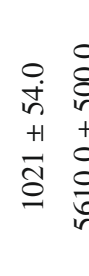 \\
\hline 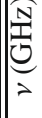 & 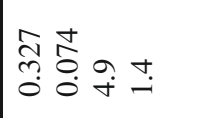 & $\underset{\substack{9 \\
\infty \\
\infty \\
0}}{0}$ & 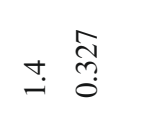 & 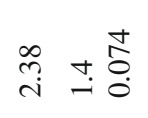 & $\underset{\infty}{+} \stackrel{+}{\stackrel{\Delta}{5}}$ & I & $\stackrel{+}{\infty} \underset{+}{+} \stackrel{+}{-}$ & $\stackrel{\&}{\infty} \underset{+}{+}$ & aे & \begin{tabular}{ll}
$\infty$ & \multirow{a}{\alpha}{} \\
$\dot{\sigma}$ & $c$
\end{tabular} \\
\hline 领 & 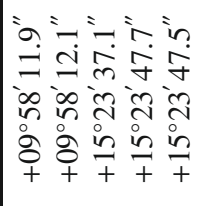 & 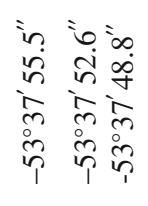 & 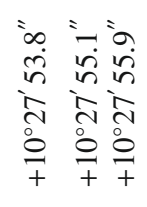 & 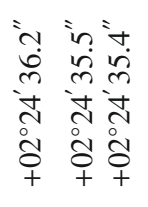 & 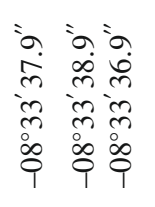 & 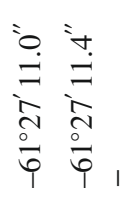 & 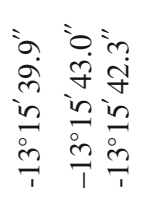 & 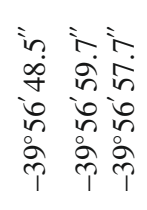 & 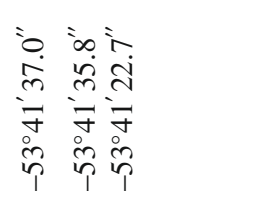 & 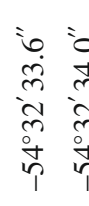 \\
\hline 8 & 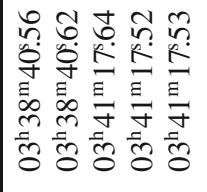 & 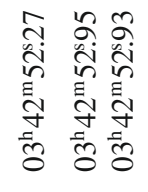 & 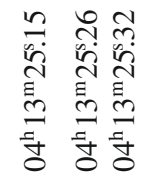 & 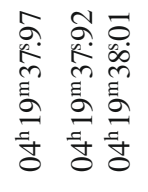 & 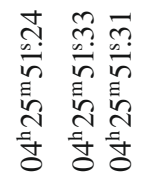 & 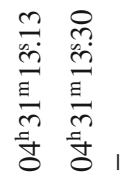 & 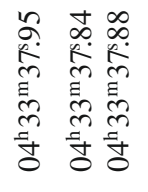 & 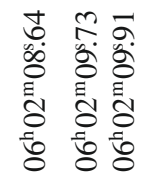 & 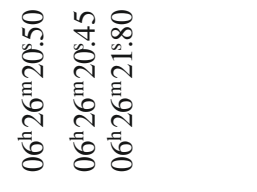 & 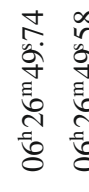 \\
\hline$\| \frac{0}{\frac{1}{8}}$ & 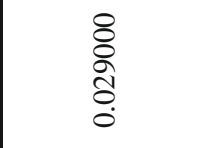 & 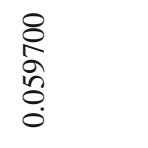 & $\begin{array}{l}8 \\
\infty \\
\infty \\
0 \\
0 \\
0\end{array}$ & 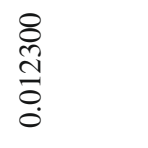 & 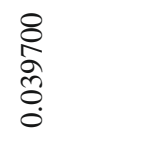 & $\begin{array}{l}8 \\
\vdots \\
\infty \\
0 \\
0 \\
0\end{array}$ & 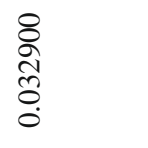 & $\begin{array}{l}8 \\
8 \\
0 \\
0 \\
0\end{array}$ & $\frac{8}{8}$ & $\frac{8}{0}$ \\
\hline 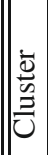 & 䓂 & $\begin{array}{l}\bar{\infty} \\
\stackrel{\infty}{n} \\
\bar{z}\end{array}$ & $\begin{array}{l}\infty \\
\stackrel{0}{8} \\
\frac{1}{4}\end{array}$ & $\begin{array}{l}0 \\
n \\
0 \\
0 \\
z\end{array}$ & 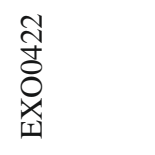 & 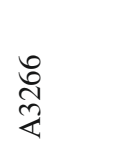 & ஓ̊ & $\begin{array}{l}\text { bे } \\
\text { ले }\end{array}$ & बू্ & 命 \\
\hline
\end{tabular}


R. Mittal et al.: AGN heating in the HIFLUGCS sample of galaxy clusters, Online Material $p 3$

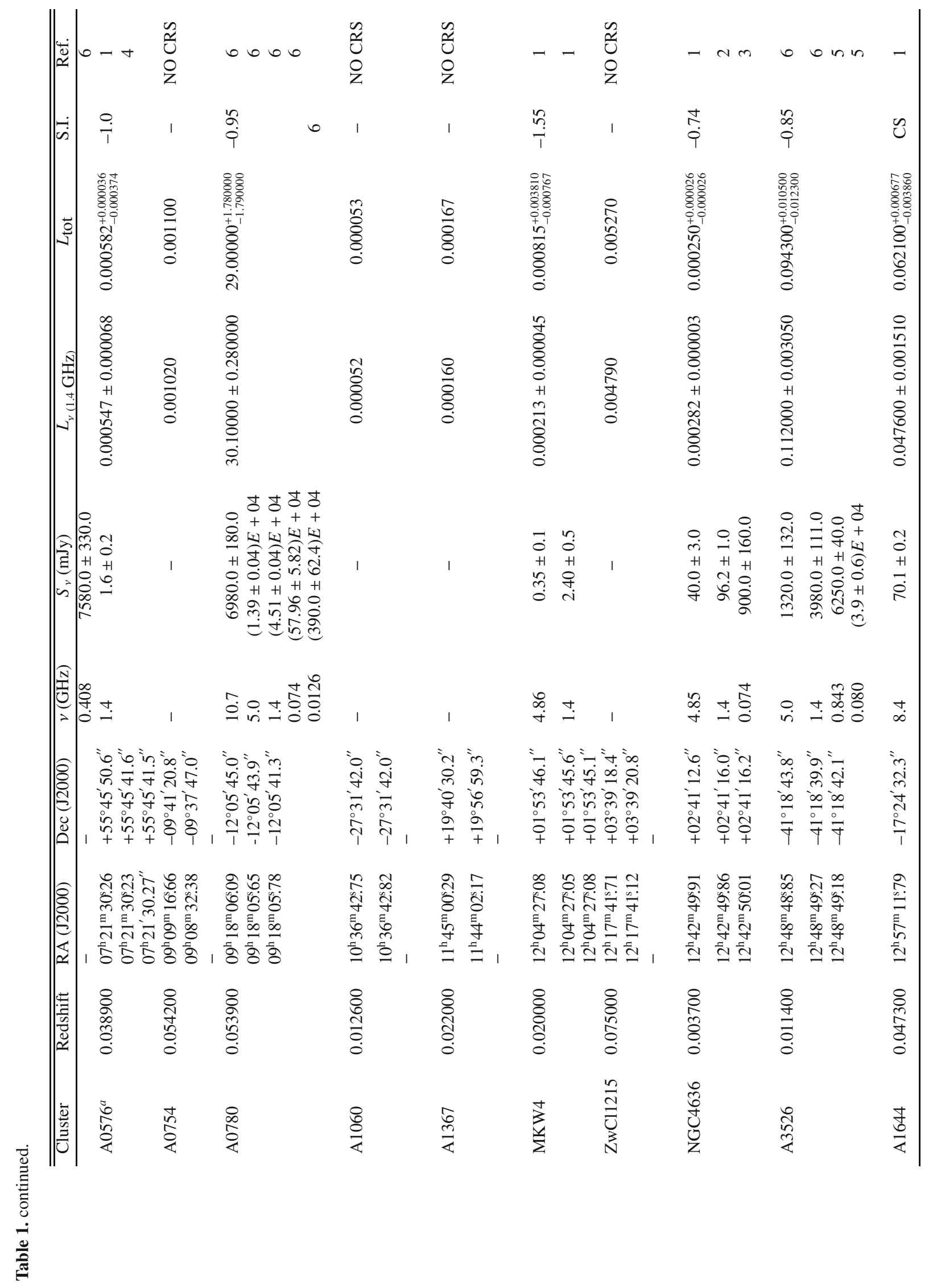


R. Mittal et al.: AGN heating in the HIFLUGCS sample of galaxy clusters, Online Material p 4

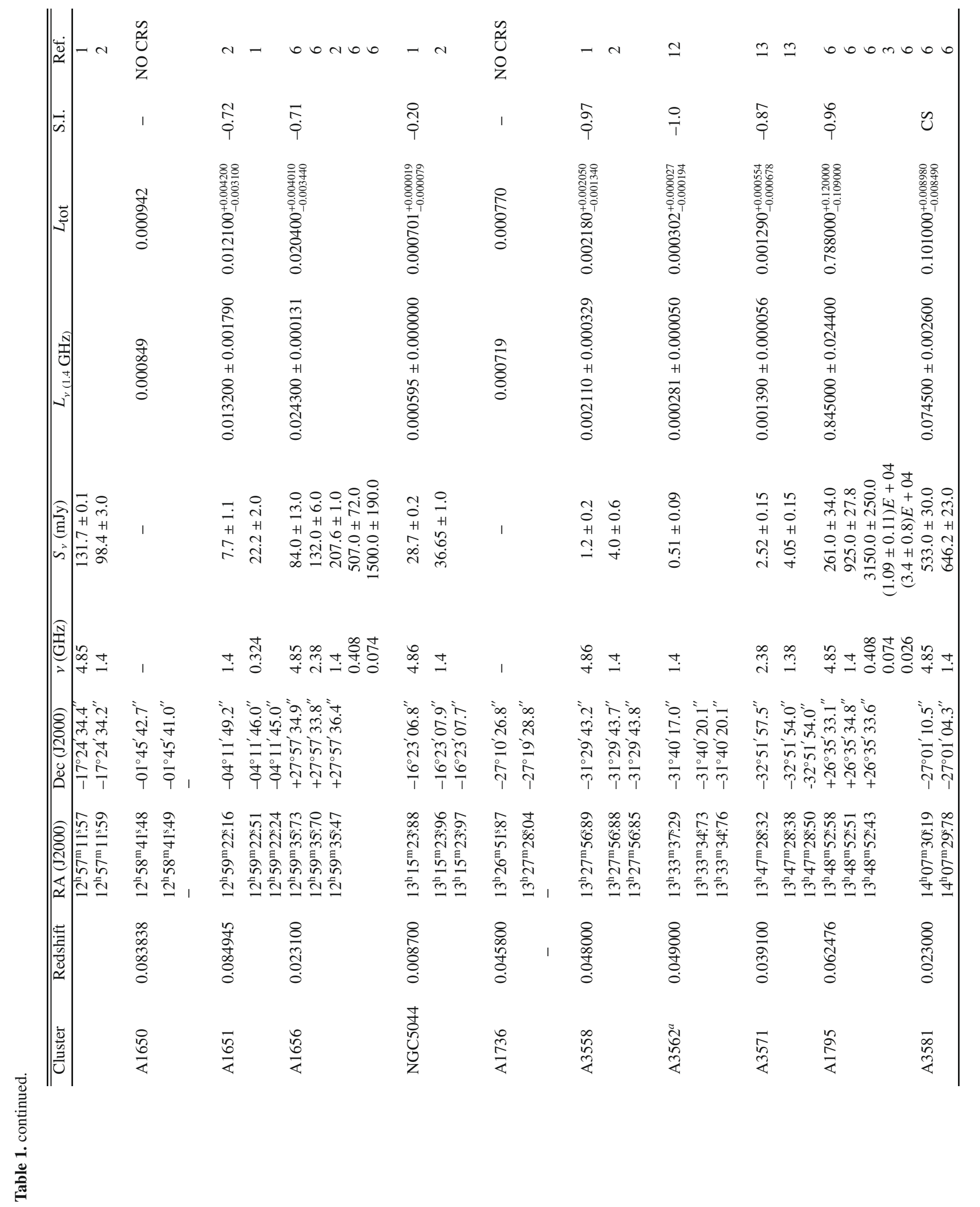


R. Mittal et al.: AGN heating in the HIFLUGCS sample of galaxy clusters, Online Material p 5

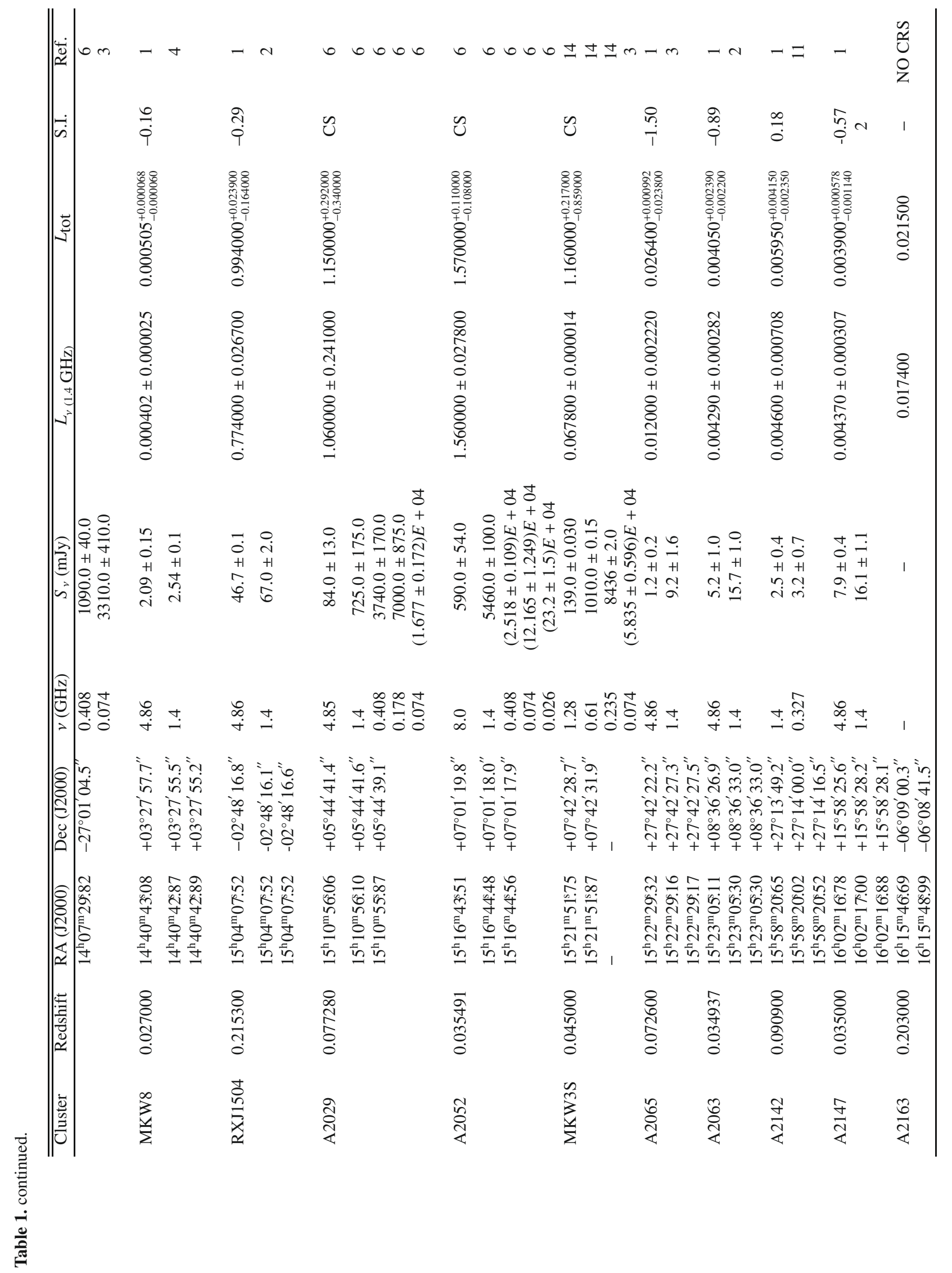




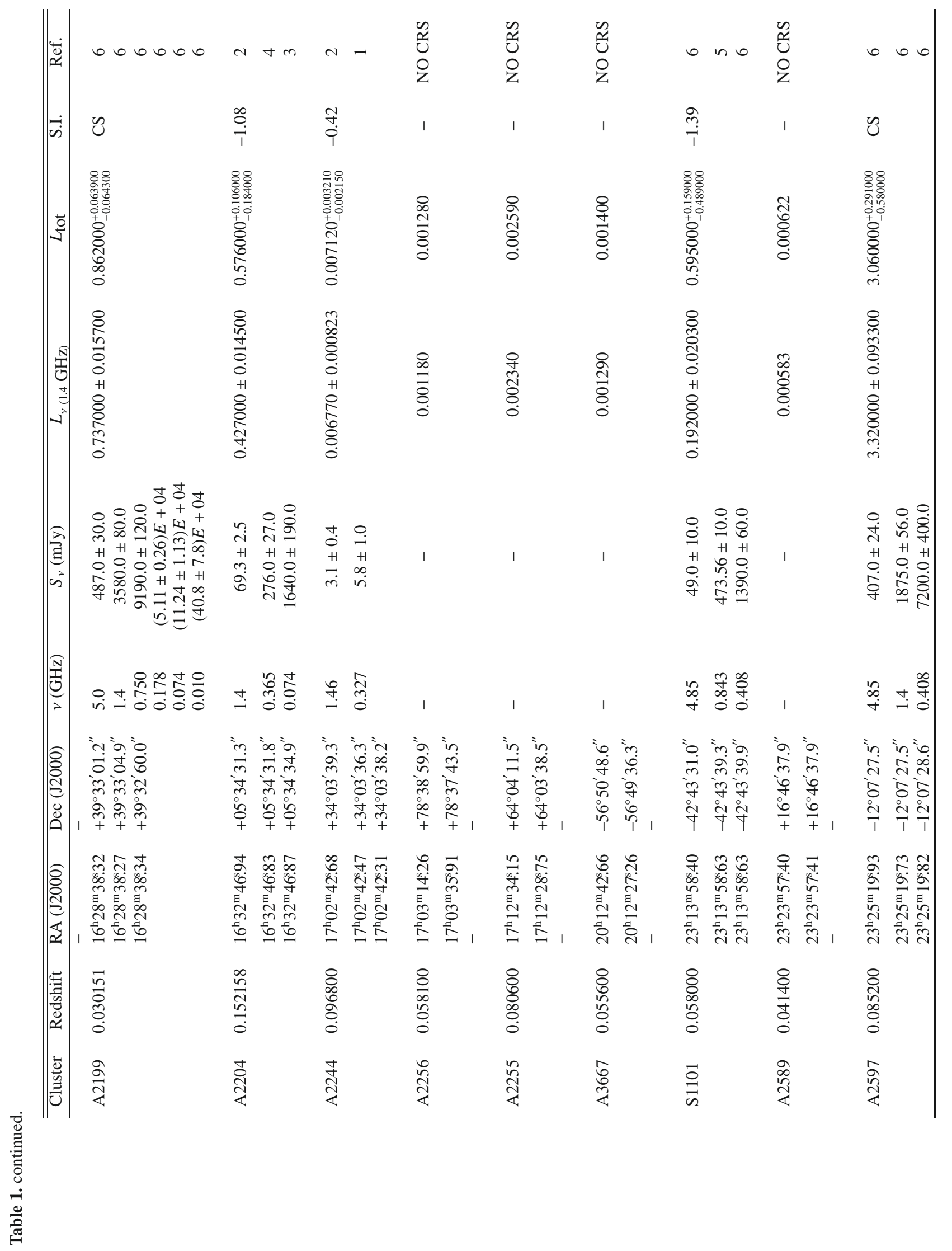


R. Mittal et al.: AGN heating in the HIFLUGCS sample of galaxy clusters, Online Material $p 7$

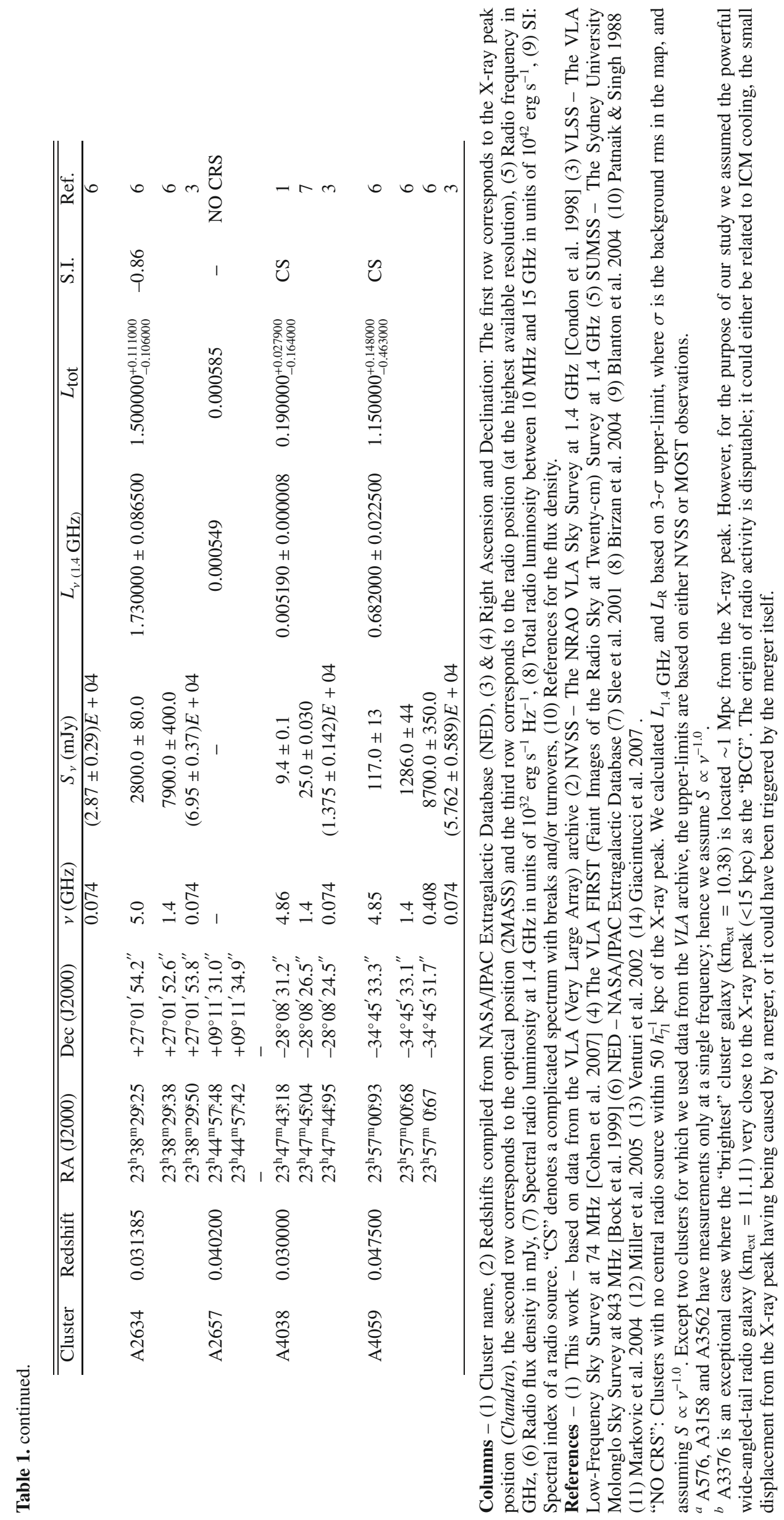

